\title{
Metal distribution in the intracluster medium: a comprehensive numerical study of twelve galaxy clusters ${ }^{\star}$
}

\author{
Harald Höller ${ }^{1}$, Josef Stöckl ${ }^{1}$, Andrew Benson ${ }^{2}$, Markus Haider ${ }^{1}$, Dominik Steinhauser ${ }^{1}$, \\ Lorenzo Lovisari ${ }^{3}$, and Florian Pranger ${ }^{1}$
}

\author{
${ }^{1}$ Institute of Astro- and Particle Physics, University of Innsbruck, Austria \\ e-mail: harald.hoeller@uibk.ac .at \\ 2 Observatories of the Carnegie Institution for Science, Pasadena, CA, USA \\ 3 Argelander-Institut für Astronomie, Bonn, Germany
}

Received 31 December 2013 / Accepted 1 July 2014

\section{ABSTRACT}

\begin{abstract}
We present a simulation setup for studying the dynamical and chemical evolution of the intracluster medium (ICM) and analyze a sample of 12 galaxy clusters that are diverse both kinetically (pre-merger, merging, virialized) and in total mass $\left(M_{\mathrm{vir}}=1.17 \times\right.$ $10^{14}-1.06 \times 10^{15} M_{\odot}$ ). We analyzed the metal mass fraction in the ICM as a function of redshift and discuss radial trends as well as projected $2 \mathrm{D}$ metallicity maps. The setup combines high mass resolution $N$-body simulations with the semi-analytical galaxy formation model GALACTICUS for consistent treatment of the subgrid physics (such as galactic winds and ram-pressure stripping) in the cosmological hydrodynamical simulations. The interface between GALACTICUS and the hydro simulation of the ICM with FLASH is discussed with respect to observations of star formation rate histories, radial star formation trends in galaxy clusters, and the metallicity at different redshifts. As a test for the robustness of the wind model, we compare three prescriptions from different approaches. For the wind model directly taken from GALACTICUS, we find mean ICM metallicities between $0.2-0.8 Z_{\odot}$ within the inner $1 \mathrm{Mpc}$ at $z=0$. The main contribution to the metal mass fraction comes from galactic winds. The outflows are efficiently mixed in the ICM, leading to a steady homogenization of metallicities until ram-pressure stripping becomes effective at low redshifts. We find a very peculiar and yet common drop in metal mass fractions within the inner $\sim 200 \mathrm{kpc}$ of the cool cores, which is due to a combination of wind suppression by outer pressure within our model and a lack of mixing after the formation of these dense regions.
\end{abstract}

Key words. hydrodynamics - methods: numerical - Galaxy: abundances - galaxies: interactions - galaxies: evolution

\section{Introduction}

The study of galaxy clusters has benefited immensely from multiwavelength studies over the past few decades. First, photometric studies of rich clusters of galaxies have revealed an increase of blue spiral galaxies with higher redshifts (Oemler 1974), which indicates that there is an evolutionary sequence in these local clusters. A few years later, the influence of the density of the environment in which galaxies reside was established as a correlation between galaxy morphology and galaxy number density (Dressler 1980). While these correlations are verifiable for relaxed galaxy clusters, kinetically complex systems pose a challenge for observational studies (see e.g. Pranger et al. 2013), which leaves questions on the kinetic state of these potentially merging or post-merger clusters open. Our best tracer for the kinetic state of these objects are radio and X-ray observations of the intracluster medium (ICM) as expounded by Sarazin (1986) and studied, for example, by Markevitch et al. (2000) and Cassano et al. (2010). In addition to the X-ray surface brightness (centroid-shift method, see, e.g., Poole et al. 2006; Maughan et al. 2008) and the presence of radio halos, the distribution of metals seems to be a valid tracer for kinetic peculiarities within galaxy clusters, which was studied observationally

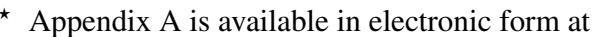
http: //www . aanda . org
}

(see, e.g., Simionescu et al. 2009) and in simulations (e.g., Kapferer et al. 2009).

A number of different processes have been suggested to be responsible for the enrichment of the ICM with metals. Rampressure (RPS) stripping (Gunn et al. 1972) is an interaction of infalling galaxies with the dense gas of cluster centers in which galactic material is removed from the gravitational potential of the galaxies and deposited into the ICM. Shortly after the discovery of iron lines in the ICM (Mitchell et al. 1976), galactic winds (De Young 1978) were suggested as a process that might be able to efficiently transport metals ICM. Mass outflow from supersonic jets as found in radio galaxies and quasars was studied numerically by De Young (1986), and observations (e.g., Wills et al. 1985) showed significant spectral Fe features associated with AGNs. While these mechanisms are of galactic origin, Gerhard et al. (2002) found an isolated compact HII region within the Virgo cluster, suggesting that there might be a fractional contribution from intracluster light to the metals found in the ICM.

In this paper we study cosmological simulations of galaxy clusters with respect to the dynamics and the chemical evolution of the ICM. We consider galactic winds and ram-pressure stripping as enrichment processes. The paper is structured as follows: in Sect. 2 we present our unique simulation setup, for which we combined a sophisticated subgrid model by including the well-tested semi-analytical model GALACTICUS into a grid 
based treatment of the ICM with the FLASH4 hydro code. The details of the interface between these two codes are described in Sect. 3, where we describe three different wind models and the ram-pressure stripping algorithm. We present the actual sample of 12 galaxy clusters we analyzed in Sect. 4. Here we also motivate the choice of objects that were included for the purpose of this work. In Sect. 5 we display and describe the results of the 12 cluster simulations as well as the comparison between the three galactic wind models depicted in Sect. 3.1. Section 6 is dedicated to a comparison of our results with observations and explores the potential of the simulation setup presented here for future studies in collaboration with X-ray surveys. We summarize the results and give a brief overview of the development roadmap in Sect. 7.

\section{Simulation setup}

Because we wish to explore feedback processes that couple galaxies and the ICM on cosmological scales, we constructed a set of consecutive simulations that incorporate cosmological structure formation, a semi-analytical galaxy formation model, and a hydrodynamical treatment of the ICM. In the following sections we present this unique setup in some detail. We note that all codes are publicly available, are well-tested within the communities, and are continuously developed and refined.

In the discussion we do not review all the implementations in detail, but focus on software and parameter choices as well as certain aspects of the software packages that are of crucial importance. For the specifics of the publicly available codes we refer to the appropriate code papers. A detailed description of the setup we have developed and assembled can be found in Höller (2014).

\subsection{IC and $\mathrm{N}$-body simulation of structure formation}

The sample of galaxy clusters depicted in Sect. 4 is generated by adopting cosmological parameter values taken from WMAP7 data (Larson et al. 2011) with the initial condition generation codes NGenIC (which is part of the GADGET-2 package, see Springel 2005) and MUSIC (Hahn \& Abel 2011). The values used are $\Omega_{\mathrm{c}}=0.266, \Omega_{\Lambda}=0.733, \Omega_{\mathrm{b}}=0.045, H_{0}=70.3$, $\sigma_{8}=0.809$ and $n_{\mathrm{s}}=0.966$ in combination with the the matter transfer function from Eisenstein \& $\mathrm{Hu}$ (1998).

For our sample we generated a large number of unconstrained cosmological volumes between $48 \mathrm{Mpc} / \mathrm{h}$ and $102 \mathrm{Mpc} / \mathrm{h}$ in size, each with $512^{3}$ dark matter particles. In these unconstrained simulations we identified galaxy clusters using a halo finder (see Sect. 2.2) and cut out a smaller volume of $20 \mathrm{Mpc} / \mathrm{h}$ with the heaviest halo in the center to facilitate handling of the data. The sample presented in Sect. 4 is a balanced mixture of different masses and kinetic states of galaxy clusters. In addition to the mass resolutions, which are just given by the relation between simulation volume and particle number, one major input parameter in the $N$-body simulations executed with GADGET-2 is the softening length $\epsilon$. In particular, test runs have shown that the number of stable, lower mass halos found is very sensitive to this parameter. We refer to Rodionov \& Sotnikova (2005), who calculated the softening needed because of the peak density in the dark matter simulation. In our case these peak densities reach up to $\sim 1.5 \times 10^{23} \mathrm{~g} / \mathrm{cm}^{3}$, yielding a (physical) value of $\epsilon \leq 0.5 \mathrm{kpc} / \mathrm{h}$. With this the values of the number of halos (and galaxies) in our simulation converged.

We output the 200 GADGET -2 snapshots equidistantly spaced in time that we use as input for the remainder of our analysis; this

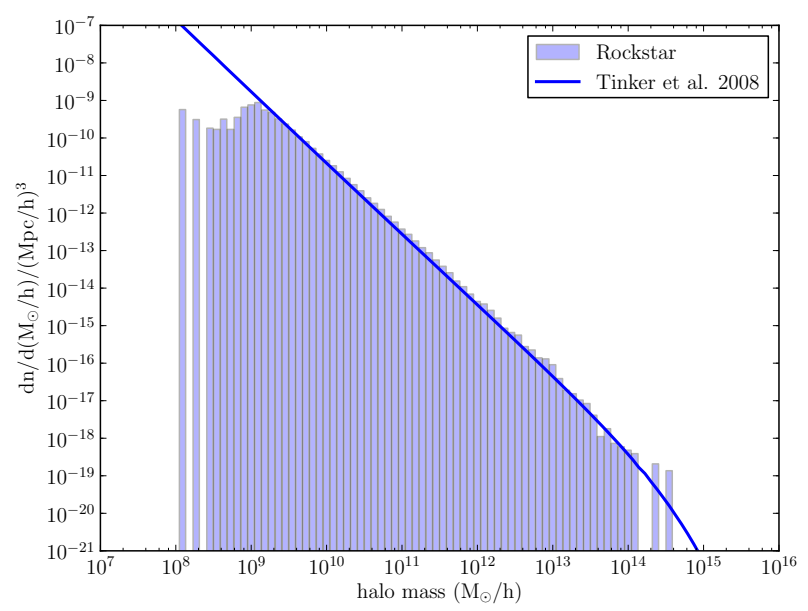

Fig. 1. Halo mass function at $z=0$ from a simulation using $512^{3}$ particles, each of mass $6.1 \times 10^{7} M_{\odot}$ (histogram), compared with the mass function of Tinker et al. (2008, line).

is already more than necessary for convergence of the galaxy properties, as shown in Benson et al. (2012).

\subsection{Halo finder and merger tree}

The identification of bound objects in the structure formation simulation is the basis for modeling galaxy properties in our semi-analytical galaxy formation model. The merger history of halos and subhalos determines the evolution of the tracked objects. To identify halos we used the software Rockstar (Behroozi et al. 2013a), which has proven very reliable in the comparison project of Knebe et al. (2011). In Fig. 1 we show the halo mass function of our highest resolution run, which yields a completeness down to $10^{9} M_{\odot}$. Merger trees were generated from Rockstar output using the algorithm Consistent Trees (Behroozi et al. 2013b), in which we enforced a mass-resolution lower limit of $\geq 1000$ particles per halo. The merger trees obtained from Consistent Trees were converted into the appropriate input format for GALACTICUS using the publicly available converter rockstar2galacticus ${ }^{1}$.

\subsection{Semi-analytical galaxy formation model}

As described in Benson (2012), GALACTICUs functions by first constructing a merger tree and then solving the set of differential equations that describe how galaxies form in that merger tree.

We constructed merger trees by simply reading their definition from the output of rockstar2galacticus. Each merger tree consists of a set of dark matter halos, some of which will be isolated halos, others of which are subhalos hosted within isolated halos. The merger-tree definition output by rockstar2galacticus specifies for each halo the descendent (i.e., the halo it becomes at the subsequent snapshot), its host (i.e., the larger halo within which it exists if it is a subhalo), and a variety of other properties, including the mass, position, velocity, dimensionless spin parameter, and half-mass radius. GALACTICUS uses this information to construct the merger tree and to initialize the masses, positions, velocities, angular momenta, and density profiles of all halos in the tree. After it is

\footnotetext{
1 https://bitbucket.org/markushaider/ rockstar2galacticus
} 


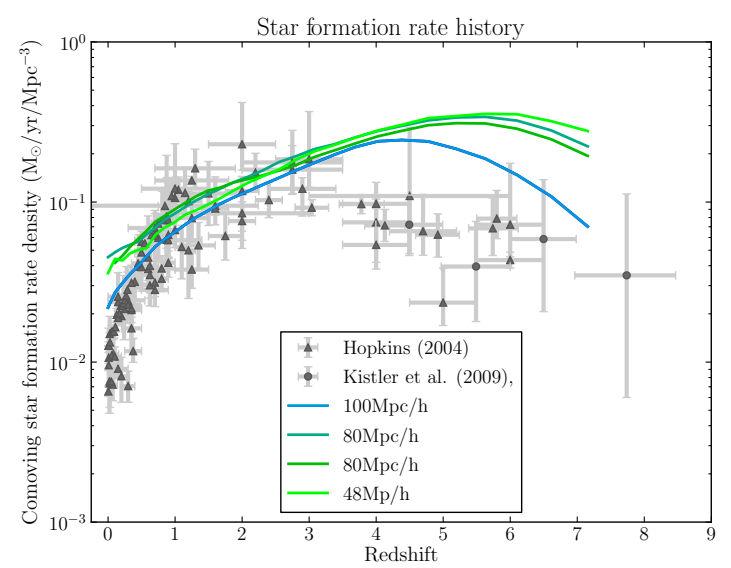

Fig. 2. Star formation histories for different simulation volumes. The larger the volumes, the better the agreement with the observational data from Hopkins (2004) and Kistler et al. (2009).

constructed, galaxy formation proceeds in this tree following GALACTICUS' usual methodology.

Merger trees extracted from $N$-body simulations require some additional considerations compared with the description of merger-tree evolution given in Benson (2012):

- Subhalos are tracked in the simulation until they fall below the detected threshold of the group-finding algorithm. This provides some information about when galaxies in subhalos should be considered to merge with another galaxy. Galacticus utilizes this information to determine both the time at which mergers will take place and the halo with which the merger occurs (including the possibility of subhalo-subhalo mergers). Since it is possible that merging will not occur immediately when the subhalo is lost from the simulation, we allowed for a delay between the time at which the subhalo was last seen and the time of merging. This delay was computed using the algorithm of Boylan-Kolchin et al. (2008), with the orbital parameters of the subhalo taken at the time at which it was last detected in the simulation.

- In merger trees derived from $N$-body simulations (as opposed to those constructed using extended Press-Schechter algorithms, for example), subhalos can sometimes escape their parent halos and again become isolated systems. GALACTICUS detects such events in merger trees and, at the appropriate time, detaches the subhalo from its host, promoting it to be again an isolated halo. This means that the halo can again begin to accrete new material from the intergalactic medium (if it continues to grow in mass).

- Another possibility in $N$-body simulation merger trees is for a subhalo to jump between branches of the tree between subsequent snapshots (i.e., the subhalo moves between host halos that are not part of the same tree branch). GALACTICUS also detects this type of event in merger trees and moves the subhalo between hosts at the appropriate time. This will affect to which halo any outflowing material from the subhalo is deposited, for example.

\subsection{Hydrodynamical simulation}

We used FLASH4 for the hydrodynamical treatment of the ICM in cosmological simulations. The Riemann solver was the unsplit HLLD implemented in FLASH4 together with a vanLeer slope limiter and Balsara prolongation for the correct treatment of the refinement boundaries. The $20 \mathrm{Mpc} / \mathrm{h}$ volume was discretized into three nested grids of $256^{3}$ cells, each of which results in a resolution of $\sim 20 \mathrm{kpc} / \mathrm{h}$ for the inner $5 \mathrm{Mpc} / \mathrm{h}$ in whose center the cluster is located at $z=0$.

Because we adopted post-processed data from the GADGET-2 dark matter simulation for the semi-analytical model and the feedback processes into the subsequent hydrodynamical simulation, we decided not to use the particle unit in FLASH4, but instead generated dark matter density fields from mapping the GADGET-2 snapshots directly onto the grid. We mapped the dark matter particles with the Delaunay tessellation field interpolation code DTFE (Schaap \& van de Weygaert 2000). The strength of this approach compared with cloud-in-cell-like algorithms is that the resolution of the grid data stays ahead of the adaptive mass resolution that is ensured by using a particle-based dark matter (DM) simulation like ours with GADGET-2. In particular, this avoids small-scale variations in the distribution of the DM particles compared with the GADGET-2 run that are caused by the grid-based restrictions on the gravitational potential and an inconsistency of the FLASH CiC formulation at refinement boundaries. Another advantage gained by employing these readin density fields is the performance improvement in FLASH4 without the particle unit.

The adjustment of the metallicities $X_{i}$ in the hydrodynamical simulation is implemented by making use of a special variable type (SPECIES) that becomes advected with the fluxes, but with the additional implication that the sum over all metal massfractions $X_{i}$ equals 1 . We initialized these SPECIES with the primordial abundances of $\mathrm{H}$ and $\mathrm{He}$ and did not model any metallicity enrichment other than galactic feedback, meaning that we did not model intracluster supernovae ( $\mathrm{SNe})$ at the current stage,

$X_{i}=\frac{\rho_{i}}{\sum \rho_{i}}, \quad \sum X_{i}=1$

We did not consistently compute the ratio of $\mathrm{H}$ and $\mathrm{He}$, but reduced its mass fraction by equal parts when enriching a cell because we are not interested in the details of $\mathrm{H} / \mathrm{He}$ abundances. For the disambiguation of enrichment that is caused by galactic winds and ram-pressure stripping (Sect. 3), we introduced distinct fields to be able to trace the evolution of either separately. At this moment we are unable to track individual elements consistently within our subgrid model GALACTICUS, hence metal mass fractions always refer to total metallicities in this work except when explicitly noted otherwise. The details of implementing the interfaces between SAM and hydrodynamical simulation can be found in Höller (2014). For the algorithms that govern the outflowed metal masses from nodes within GALACTICUS, we refer to Benson $(2012,2010)$.

Cooling is one physical process that is directly affected by the enrichment of the ICM with heavier elements from the subgrid physics. We include cooling tables from Sutherland \& Dopita (1993), which are given for a number of temperatures and metallicities between which we linearly interpolated in logspace. The tables include cooling functions over a range of $10^{4}-10^{8.5} \mathrm{~K}$, but we truncated the cooling below $T=10^{6} \mathrm{~K}$ to avoid artificially dense and cool regions during the formation process of the galaxy cluster. This lower cooling limit is not even remotely reached when the actual merger-shock-heated cores have formed in the simulations. Moreover, we detected problematic behavior only in a run with the highly effective wind model B, which we describe in Sect. 3.1. Observations of cooling flows (Fabian 1994) indicate that their occurrence is not only related to the kinetic state of the galaxy cluster and additional heating processes such as AGN feedback (Fabian 2012), but also 
to the ICM metallicity (Allen \& Fabian 1998). In Sect. 5 we discuss the finding of cool cores in our simulations with respect to the ICM metallicities.

\section{Feedback models}

The main strength of our setup is the ability to model galactic feedback processes in a cosmological simulation for a large number of objects (typically $\sim 10^{4}$ ). We treat the input of mass, momentum and energy from galactic winds and ram-pressure stripping into the ICM by adopting numerical and analytical prescriptions. In the following sections we discuss the details of these feedback models.

\subsection{Galactic winds}

The details of mass, momentum, and energy outflow of galactic winds is observationally poorly constrained because of the extremely low densities and high temperatures of these flows. However, there is consensus that starburst winds, driven by kinetic energy and momentum from stellar winds and supernovae, play a major role not only in the evolution of galaxies themselves - as shown in numerical studies such as Cole et al. (2002) or Springel \& Hernquist (2003) - but especially for the enrichment of the gas in the Universe with heavier elements (Adelberger et al. 2003; Steidel et al. 2010). Multiwavelength observations in the optical (e.g., Heckman et al. 1990), UV (Meurer et al. 1995; Gonzalez Delgado et al. 1998) and X-ray bands (Su et al. 2010; Strickland \& Heckman 2009) have indicated that there is a strong correlation between the strengths of starbursts and kinematically confirmed galactic outflows or diffuse ionized gas in the close vicinity of these galaxies.

To model galactic wind feedback from the GALACTICUS output into the ICM of the hydrodynamical simulation, we adapted two models derived from numerical studies. For the first model (model A) we refer to Leitherer et al. (1999) and Veilleux et al. (2005), who presented a very simple wind prescription derived from simulations with Starburst99. This model yields a constant mass-loading factor for solar metallicity and constant outflow and mechanical luminosity integrated over 40 Myr scales,

$$
\begin{aligned}
& \dot{M}_{* \mathrm{~A}}=0.26\left[S F R / M_{\odot} \mathrm{yr}^{-1}\right] M_{\odot} \mathrm{yr}^{-1} \\
& \dot{E}_{* \mathrm{~A}}=7 \times 10^{41}\left[S F R / M_{\odot} \mathrm{yr}^{-1}\right] \mathrm{erg} \mathrm{s}^{-1} \\
& \dot{p}_{* \mathrm{~A}}=5 \times 10^{33}\left[S F R / M_{\odot} \mathrm{yr}^{-1}\right] \text { dyne }
\end{aligned}
$$

The expected residing time within a grid cell of a galaxy with typical $\sim 1000 \mathrm{~km} \mathrm{~s}^{-1} \cong 10^{-6} \mathrm{kpc} / \mathrm{yr}$ velocity in the innermost of our grids is $\geq 20$ Myr. Therefore, for the vast majority of objects, not more than one cell is occupied during this 40 Myr integration time. Because the resolution of the spatial grid is not high enough to resolve galactic morphologies (and orientations), we added mass, momentum, and pressure from Eq. (2) cell-wise. Implicitly, therefore, we assumed that the outflowed material mixes and equilibrates with the surrounding ICM on the time scales mentioned above. This wind model does not provide a parametrization with the galaxies' masses. From analytical and numerical analysis we know that the gravitational binding energy of the host strongly influences the efficiency of the galactic outflow. We adopted the numerical results from Recchi \& Hensler (2013), which imply an averaged wind efficiency as a function of the total baryonic content of the galaxy $M_{\mathrm{b}}$,

$$
\mathrm{Eff}_{\mathrm{A}}=\left\{\begin{array}{lll}
1 & \text { for } & \log \left(M_{\mathrm{b}}\right)<7 \\
3.8-0.41 \log \left(M_{\mathrm{b}}\right) & \text { for } 7 \leq \log \left(M_{\mathrm{b}}\right) \leq 9.4, \\
0 & \text { for } \log \left(M_{\mathrm{b}}\right)>9.4
\end{array}\right.
$$

The mass-loading factor of constant 1 for objects of mass $\log \left(M_{\mathrm{b}}\right)<7$ most likely underestimates the actual wind in this range, but the mass-resolution limit of our SAM is approached in this same regime. A second efficiency parameter describes the pressure contrast of the wind with respect to the outer confining pressure of the ICM. Currently, we only consider isotropic gas pressure for the wind suppression and no ram pressure from velocity differences between the infalling galaxies and the ICM. When the ICM pressure exceeded the wind pressure that we obtain from Eq. (2), the flux from the galaxies into the ICM was set to zero. We also adopted from Recchi \& Hensler (2013) the resulting metallicity of the galactic outflow with constant solar value $Z_{\odot}$, for which we refer to Lodders (2003) with a value of $Z_{\odot}=0.0149$.

The second prescription implemented (model B) is taken from Hopkins et al. (2012), which is another systematic study on galactic winds making use of Starburst99. Their results show a sublinear relation between star formation rate and mass flux and a wind efficiency with steeper dependency at a higher level for less massive galaxies,

$\dot{M}_{* \mathrm{~B}}=3.0\left[S F R / M_{\odot} \mathrm{yr}^{-1}\right]^{0.7} M_{\odot} \mathrm{yr}^{-1}$.

Because this wind model does not provide for energy and momentum fluxes, we calculated the internal energy of the outflowed material for a $10^{7} \mathrm{~K}$ gas with $\dot{E}_{* \mathrm{~B}}=10^{7} k_{\mathrm{B}}(\gamma-1)$ and a corresponding gas pressure of $\dot{p}_{* \mathrm{~B}}=\sqrt{2 \dot{M}_{* \mathrm{~B}} \dot{E}_{* \mathrm{~B}}}$, assuming an ideal gas with $\gamma=5 / 3$ :

$\mathrm{Eff}_{\mathrm{B}}=32.2-2.6 \log \left(M_{\mathrm{b}}\right)$.

In addition, we added the pressure contrast efficiency to this prescription to take into account wind suppression in high-density regimes at the galaxy cluster centers.

Below we introduce a variation of this as model B', where the wind efficiency is set to be equivalent to model A:

Eff $_{\mathrm{B}^{\prime}} \equiv$ Eff $_{\mathrm{A}}$.

The galactic wind tracer of GALACTICUS taken directly from the semi-analytical model composes our model C. GALACTICUS tracks gas that has been ejected from galaxies, but has yet to reequilibrate and become part of the hydrostatic hot atmosphere surrounding each galaxy. We refer to this material as outflowed gas. The evolution of the mass of this component is given by

$$
\begin{aligned}
\dot{M}_{\text {outflowed }}= & \beta_{\text {disk }} \dot{M}_{\star \text {,disk }}+\beta_{\text {spheroid }} \dot{M}_{\star, \text { spheroid }} \\
& -\delta M_{\text {outflowed }} / \tau_{\text {dyn }} .
\end{aligned}
$$

Here, the first two terms describe supernovae-driven outflows from the disk and spheroid components of galaxies. These outflow rates are proportional to the star formation rates, $\dot{M}_{\star}$, with the coefficient of proportionality given by

$\beta=\left(V_{\text {outflow }} / V\right)^{\alpha_{\text {outflow }}}$,

where $V$ is the circular velocity of the component at its scale radius, and we adopted $V_{\text {outflow }}=250 \mathrm{~km} \mathrm{~s}^{-1}$ and $100 \mathrm{~km} \mathrm{~s}^{-1}$ for disk and spheroid, and $\alpha_{\text {outflow }}=3.5$ for both components. The final term in Eq. (7) describes the re-incorporation of the 
outflowed material into the hydrostatic hot atmosphere. Here, $\tau_{\text {dyn }}$ is the dynamical time of the halo, and $\delta=5$.

Because we introduced source terms into the equations of mass, momentum, and energy conservation, our numerical scheme is no longer strictly conservative. Therefore we limited the density, pressure, and internal energy contrast per time step to $1 \%$ of the cell values for stability reasons. In addition to the numerical motivation, the recycling of matter via the subgrid model is not perfectly consistent because we did not model accretion of mass onto the galaxies in form of negative source terms. For studying ICM metal enrichment at the current grid resolution, however, the details of this interface dynamics are not relevant.

\subsection{Ram-pressure stripping}

Ram-pressure stripping in galaxy clusters contributes not only to the surpression of galactic star formation rates (SFRs) as a strong function of cluster-centric radius, but also deposits partially enriched material into the ICM along the infalling trajectories, as shown in surveys by Lewis et al. (2002) and numerical simulations by Quilis et al. (2000), for instance. Both effects have to be taken into account for our study of the metallicity distribution in galaxy clusters. With an SFR density interpretation of the morphology density relation (Gunn et al. 1972) in galaxy clusters, the suppression of galactic winds will not only be caused by external pressure, but also by an ever-decreasing intrinsic SFR. The contribution from these supernovae and stellar-winddriven outflows will decrease with cluster-centric radius, while ram-pressure stripping is acting in dense environments and high relative velocities, as we mainly find inside the virial radii of galaxy clusters.

To model galactic disk ram-pressure stripping we applied the criterion of Gunn\&Gott (Gunn et al. 1972) by comparing the disk-restoring force at a given radius with the ram pressure exerted on the galaxy moving through the ICM:

$p_{\text {ram }}=\rho_{\mathrm{ICM}} v_{\text {rel }}^{2}$.

The surface densities $\sigma_{\text {gas }}$ and $\sigma_{\text {stellar }}$ are taken directly from the GALACTICUS models.

$F_{\text {rest }}(r)=2 \pi G \sigma_{\text {gas }}(r) \sigma_{\text {stellar }}(r)$.

Galactic disks in GALACTICUS are modeled as having exponential surface density profiles. Because the density decreases monotonically, we therefore simply iterated over radius and stripped all material outside the stripping radius $r_{\text {strip }}$ where $p_{\text {ram }}>F_{\text {rest }}(r)$. The material lost thus amounts to

$M_{\text {strip }}=\int_{r_{\text {strip }}}^{\infty} r \frac{\mathrm{e}^{\left(-r / r_{\text {disk }}\right)}}{r_{\text {disk }}^{2}} \mathrm{~d} r=\frac{\left(r_{\text {strip }}+r_{\text {disk }}\right)}{r_{\text {disk }}} M_{\text {disk }} \mathrm{e}^{\left(-r_{\text {strip }} / r_{\text {disk }}\right)}$.

Because ram-pressure stripping is not an instantaneous effect, we determined the actual fluxes into the ICM by defining a ram-pressure stripping time scale that we took from Steinhauser et al. (2012), who found stripping timescales between 100 and 250 Myr. For the total amount of gas and metals stripped, and thus deposited into the ICM, the exact number of this time scale is not very relevant because the trajectories of infalling galaxies will result in much longer paths, along which they experience ram pressure.

At this point of our setup development we did not model the effect that disk ram-pressure stripping has on the galaxies.
However, we are working on an implementation where we take this process into account by directly feeding information about the ram-pressure force extracted from the hydrodynamical simulation into GALACTICUS. In FLASH we keep track of the lost material on a per-galaxy basis. This mass of lost material is allowed to decrease on the free-fall time scale of the halo, allowing for re-accretion of gas for objects that were stripped before (see, e.g., Benson \& Bower 2011). Our approach furthermore assumes that galactic disks do not react to the outer stripping by radial pressure equilibration. In other words, the stripping radius is a sharp outer boundary and the disk can only grow beyond this radius via accretion.

The metallicity profiles of the disks were modeled using results taken from Ferguson et al. (1998), where the mean O/H ratio is given by

$\log (\mathrm{O} / \mathrm{H})=-2.8-0.65 \log \left(R_{25}\right)$.

For the reference solar abundance ration of $\mathrm{O} / \mathrm{H}=0.429$ we refer to Asplund et al. (2009). It is probably a fairly crude assumption to model all disks with log-linear profiles, because observations have shown a wide variety of radial metallicity gradients (see, e.g., Shaver et al. 1983; Zaritsky et al. 1994). Particularly in more recent works (Bresolin et al. 2012; Scarano \& Lépine 2013) observations give remarkable evidence for an omnipresent break at the corotation radius, which suggests that a log-linear profile might be underestimating the amount of metals in the outer parts of galactic disks.

\section{Galaxy cluster sample}

Our sample of 12 clusters was selected in such a way as to permit an analysis across a range of masses and states of virialization. In Table 1 we present the main parameters used for generating the initial conditions as well as the main characteristics of the clusters, such as $r_{200}$ and $M_{200}$. The differences in the virialized masses of the most massive halos, the brightest cluster galaxies (BCG) $M_{\text {vir,BCG }}$ and the $M_{200}$ values are first indicators of the degree of virialization of these objects. The difference in mass resolutions is solely caused by the differences in volumes of the initial $N$-body simulations. We used the same cosmological parameters and number of DM particles for all runs as described in Sect. 2.1.

The first and foremost physical quantity that we require to model the galactic winds and the enrichment of the ICM are the SFRs. In Fig. 3 we plot the SFR histories of all 12 clusters. It is clear that not only can the integrated SFRs differ enormously between the least massive clusters (e.g., C05) and more massive ones (such as C01 or C02), but that the SFR at $z=0$ can also differ by almost an order of magnitude. In Sect. 5 we discuss the structure formation histories of these objects also with respect to the relative peaks in the SFR history curves. In many cases, these peaks directly correspond to merger events. To interpret the plot, we recall that the $20 \mathrm{Mpc} / \mathrm{h}$ volumes are not directly comparable with the observational data because we derived $\Omega_{\mathrm{c}}$ values inside these boxes between $\Omega_{\mathrm{c}} \sim 0.7-0.9$, and in this respect these volumes are no longer representative for the cosmos. Specifically, they are highly overdense regions by virtue of having been selected to contain massive clusters. One direct consequence of this volume cut is that the SFRs plotted are significantly higher than the observational comparison data from Hopkins (2004) and Kistler et al. (2009). This tendency is also shown in Fig. 2, we show that the degree of accordance with observational data increases with simulation volume. 
Table 1. Cluster sample.

\begin{tabular}{lccccccccc}
\hline \hline Cluster & IC gen & $\begin{array}{c}\text { Box } \\
{[\mathrm{Mpc} / \mathrm{h}]}\end{array}$ & Seed & $\begin{array}{c}\text { Cutout center } \\
x-y-z[\mathrm{Mpc} / \mathrm{h}]\end{array}$ & $\begin{array}{c}M_{\text {vir,BCG }} \\
{\left[M_{\odot}\right]}\end{array}$ & $\begin{array}{c}R_{\text {vir,BCG }} \\
{[\mathrm{Mpc} / \mathrm{h}]}\end{array}$ & $\begin{array}{c}\text { DM mass res } \\
{\left[M_{\odot}\right]}\end{array}$ & $\begin{array}{c}r_{200} \\
{[\mathrm{Mpc} / \mathrm{h}]}\end{array}$ & $\begin{array}{c}M_{200} \\
{\left[M_{\odot}\right]}\end{array}$ \\
\hline C01 & MUSIC & 80 & 13671 & $38-47-66$ & $5.44 \mathrm{E} 14$ & 2.39 & $2.82 \mathrm{E} 8$ & 1.24 & $4.37 \mathrm{E} 14$ \\
C02 & MUSIC & 80 & 30596 & $21-16-64$ & $1.06 \mathrm{E} 15$ & 2.99 & $2.82 \mathrm{E} 8$ & 1.48 & $7.54 \mathrm{E} 14$ \\
C03 & MUSIC & 80 & 30596 & $22-16-62$ & $2.09 \mathrm{E} 14$ & 1.53 & $2.82 \mathrm{E} 8$ & 0.99 & $2.24 \mathrm{E} 14$ \\
C04 & MUSIC & 48 & 1502 & $35-33-20$ & $2.99 \mathrm{E} 14$ & 1.90 & $6.1 \mathrm{E} 7$ & 0.92 & $1.81 \mathrm{E} 14$ \\
C05 & MUSIC & 80 & 7964 & $62-62-39$ & $1.17 \mathrm{E} 14$ & 1.44 & $2.82 \mathrm{E} 8$ & 0.73 & $8.7 \mathrm{E} 13$ \\
C06 & MUSIC & 64 & 22109833 & $33-16-11$ & $2.08 \mathrm{E} 14$ & 1.75 & $9.7 \mathrm{E} 7$ & 0.87 & $1.57 \mathrm{E} 14$ \\
C07 & NGenIC & 100 & 27036 & $89-49-70$ & $1.45 \mathrm{E} 14$ & 1.71 & $5.52 \mathrm{E} 8$ & 0.79 & $1.13 \mathrm{E} 14$ \\
C08 & MUSIC & 80 & 17794 & $25-29-42$ & $2.69 \mathrm{E} 14$ & 1.89 & $2.82 \mathrm{E} 8$ & 0.95 & $1.99 \mathrm{E} 14$ \\
C09 & MUSIC & 80 & 25922 & $25-48-52$ & $5.29 \mathrm{E} 14$ & 2.37 & $2.82 \mathrm{E} 8$ & 1.24 & $4.36 \mathrm{E} 14$ \\
C10 & MUSIC & 80 & 32706 & $66-39-23$ & $5.45 \mathrm{E} 14$ & 2.41 & $2.82 \mathrm{E} 8$ & 1.23 & $4.26 \mathrm{E} 14$ \\
C11 & MUSIC & 48 & 8736 & $19-14-24$ & $2.11 \mathrm{E} 14$ & 1.79 & $6.1 \mathrm{E} 7$ & 0.90 & $1.69 \mathrm{E} 14$ \\
C12 & NGenIC & 100 & 20905 & $34-76-35$ & $2.49 \mathrm{E} 14$ & 1.86 & $5.52 \mathrm{E} 8$ & 0.98 & $2.15 \mathrm{E} 14$ \\
\hline
\end{tabular}

Notes. For traceability we list the initial conditions generators (Col. 2), the box sizes (Col. 3), and the random seeds (Col. 4). With the cosmological parameters listed in Sect. 2.1 it is possible to reproduce the $N$-body simulations. We specify the (approximate) position of the clusters on which we centered the $20 \mathrm{Mpc} / \mathrm{h}$ cutout in Col. 5 .

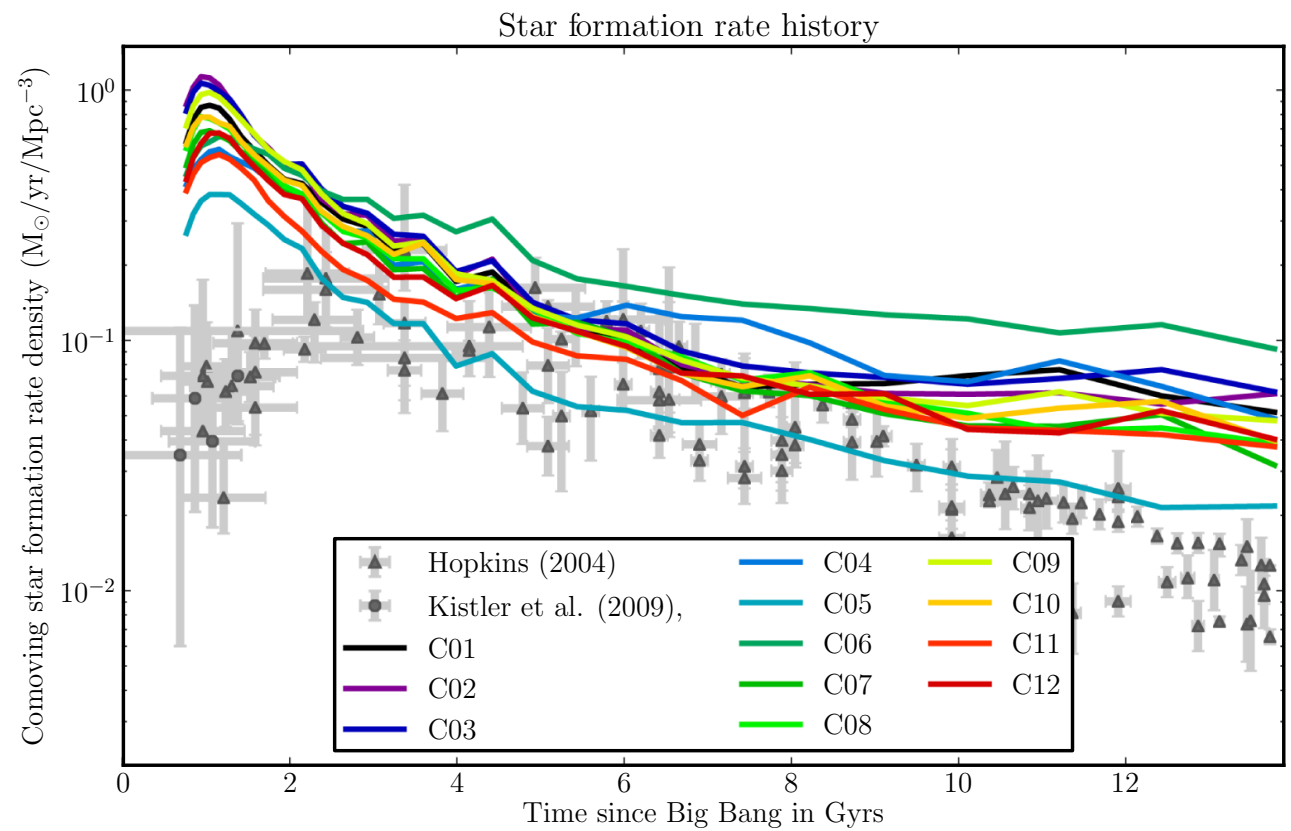

Fig. 3. SFR histories of all 12 clusters in Gyr.

Furthermore, GALACTICUS probably overestimates the SFRs at low redshifts, especially within the virial radius of the galaxy clusters, because of the lack of a generic treatment of disk rampressure stripping.

In Fig. 5 we plot the radially binned mean SFRs of all samples of galaxies and our disky galaxies, which we define as those with a nonzero disk mass. The quenching of the SFR inside the virial radius is mainly due to the hot-halo ram-pressure stripping. This is modeled in Galacticus using the results from Font et al. (2008) and affects all halos that are subhalos of another halo. Within the BCG virial radius, all infalling galaxies are satellites and hence experience stripping of their hot halo while falling into the galaxy cluster. The radially binned mean velocities are plotted in Fig. 6 and support the expected increase in efficiency of ram-pressure stripping with decreasing cluster centric radius.

In Fig. 4 we illustrate the different evolution of diskdominated (DD) and spheroid-dominated (SD) galaxies in our simulations. The classification of these two types is straightforward. We define a bulge-to-total-mass ration $B / T$ :

$B / T=\frac{M_{\star, \mathrm{sph}}+M_{\mathrm{gas}, \mathrm{sph}}}{M_{\star, \mathrm{sph}}+M_{\mathrm{gas}, \mathrm{sph}}+M_{\star, \text { disk }}+M_{\mathrm{gas}, \text { disk }}}$,

and identify $B / T \leq 0.5$ as disk-dominated (DD) and $B / T>0.5$ as spheroid-dominated (SD), while the morphological classification is based on the plotted time. Our data clearly show that disky galaxies primarily contribute to metals ejected and mixed into the ICM. This seems to contradict Arnaud et al. (1992), who found for a sample of local galaxy clusters a correlation between total cluster gas mass (and iron mass) and luminosity of ellipticals and S0s, but no correlation between cluster gas mass and spiral luminosity. The authors concluded that the iron mass in the ICM is probably directly proportional to the stellar mass in cluster ellipticals and S0s, and that therefore only ellipticals were involved in the metal enrichment of the ICM. In our simulation data the gas mass as function of galaxy luminosity and morphology agrees perfectly with the results presented 
H. Höller et al.: The distribution of metals in the ICM

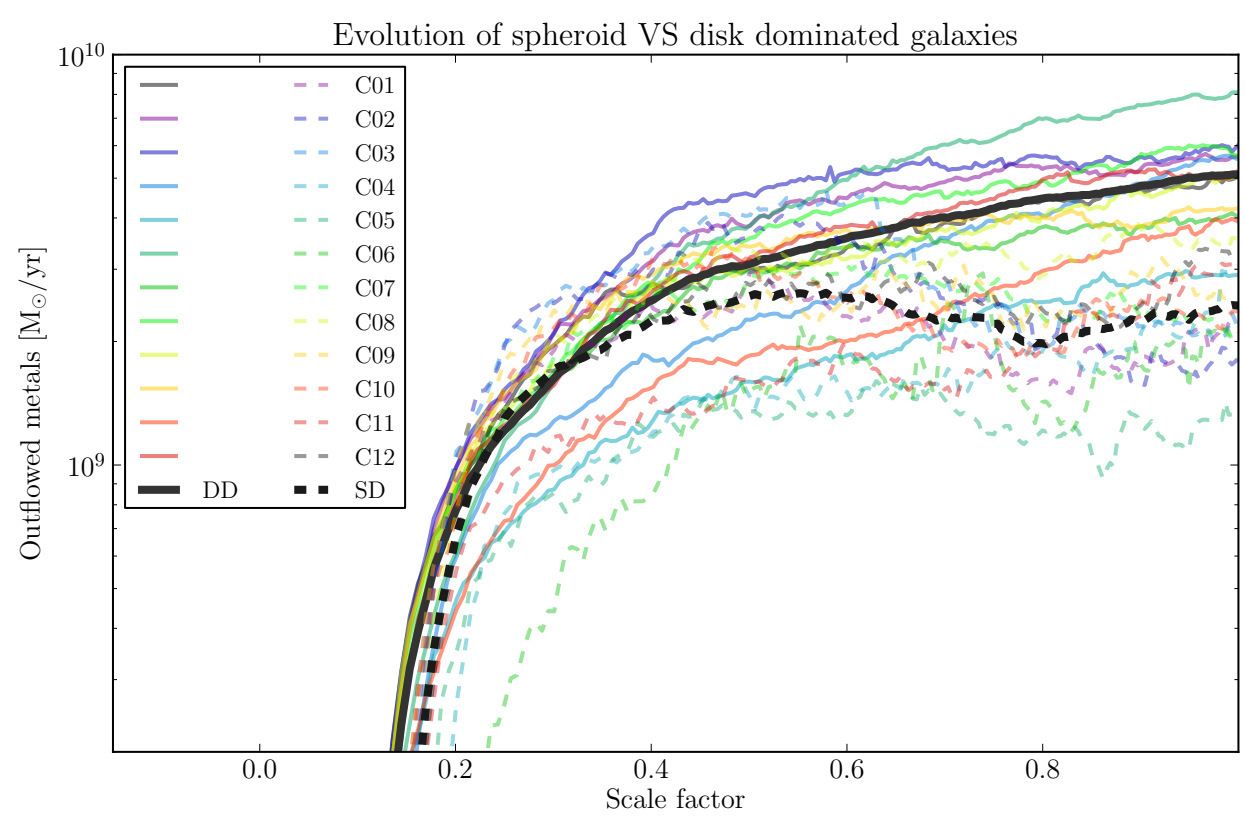

Fig. 4. Evolution of spheroid-dominated (SD, dotted) and disk-domitaned (DD, solid lines) galaxies with respect to the outflowed metals. The thicker, dark lines denote the mean values of all 12 clusters.

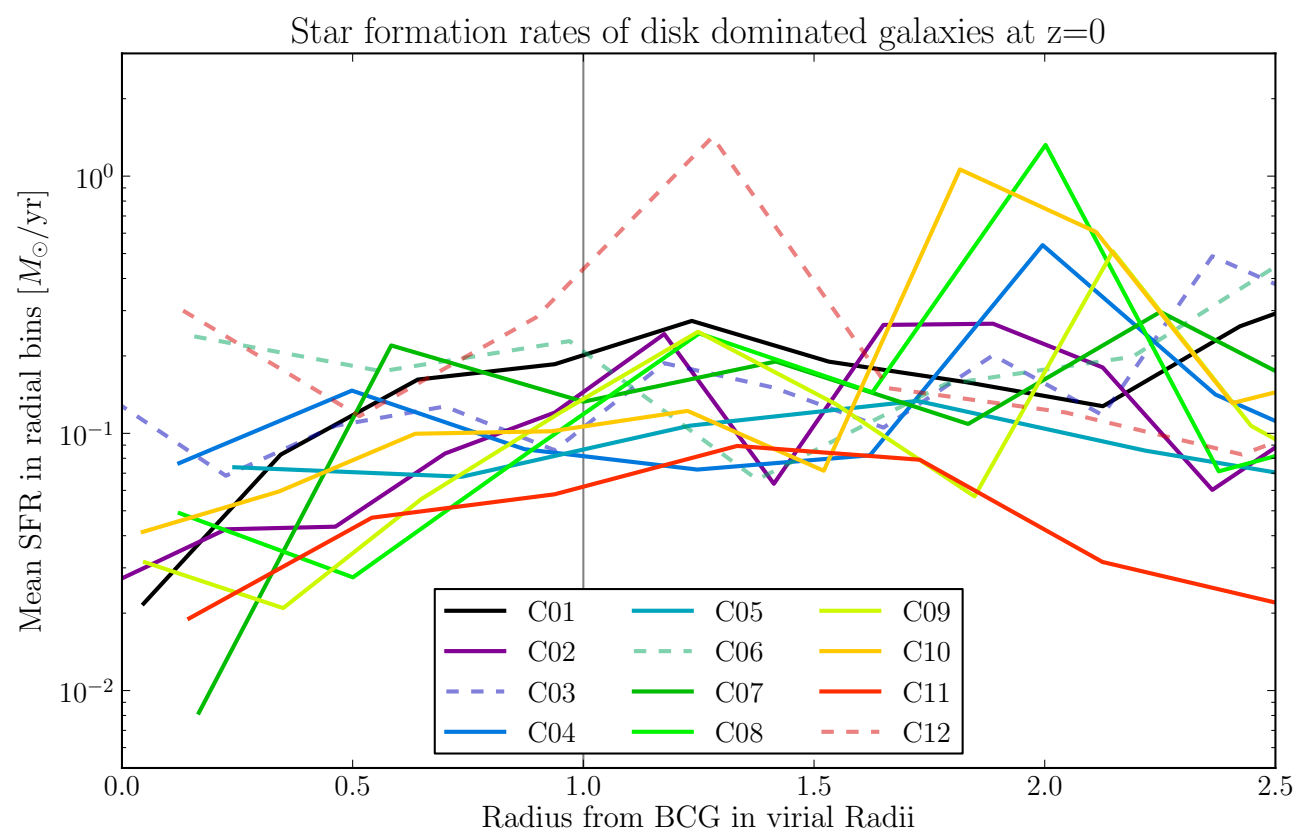

Fig. 5. Radially binned mean star formation rates from GALACTicus. The dotted, semitransparent lines belong to clusters that are in the least virialized states (see Sect. 5).

in Arnaud et al. (1992), see Table 2. However, from that finding we do not deduce that only ellipticals (and SOs) were involved in metal enrichment, but refer to the generally established idea of a morphological galaxy transformation in clusters (see, e.g., Vogt et al. 2004). Spiral galaxies that contributed to the ICM metal content in the past might have been transformed and, at the present epoch, be classified as S0s or ellipticals.

We discuss the necessity of generating and analyzing samples that are both narrower in kinetic states and larger in number for statistically significant statements in Sect. 7. The 12 clusters presented here illustrate the potential of the simulation setup and the wide spectrum of objects that can be covered and studied, however.
Table 2. Comparison of simualtion results with data from Arnaud et al. (1992) within the inner $3 \mathrm{Mpc}$ of the galaxy clusters.

\begin{tabular}{lll}
\hline \hline & Observational & Simulation sample \\
\hline Gas mass and SD & $1.1 \pm 0.25(\mathrm{E})$ & $1.19(B / T \geq 0.6)$ \\
& $1.5 \pm 0.25(\mathrm{E}+\mathrm{S} 0)$ & $1.26(B / T \geq 0.5)$ \\
Metal mass and SD & $1.0(\mathrm{E}+\mathrm{S} 0)$ & 1.13 \\
\hline
\end{tabular}

\section{Results of cosmological hydrodynamical cluster simulations}

The results presented in the subsections below are based on simulations made with wind model $\mathrm{C}$. 


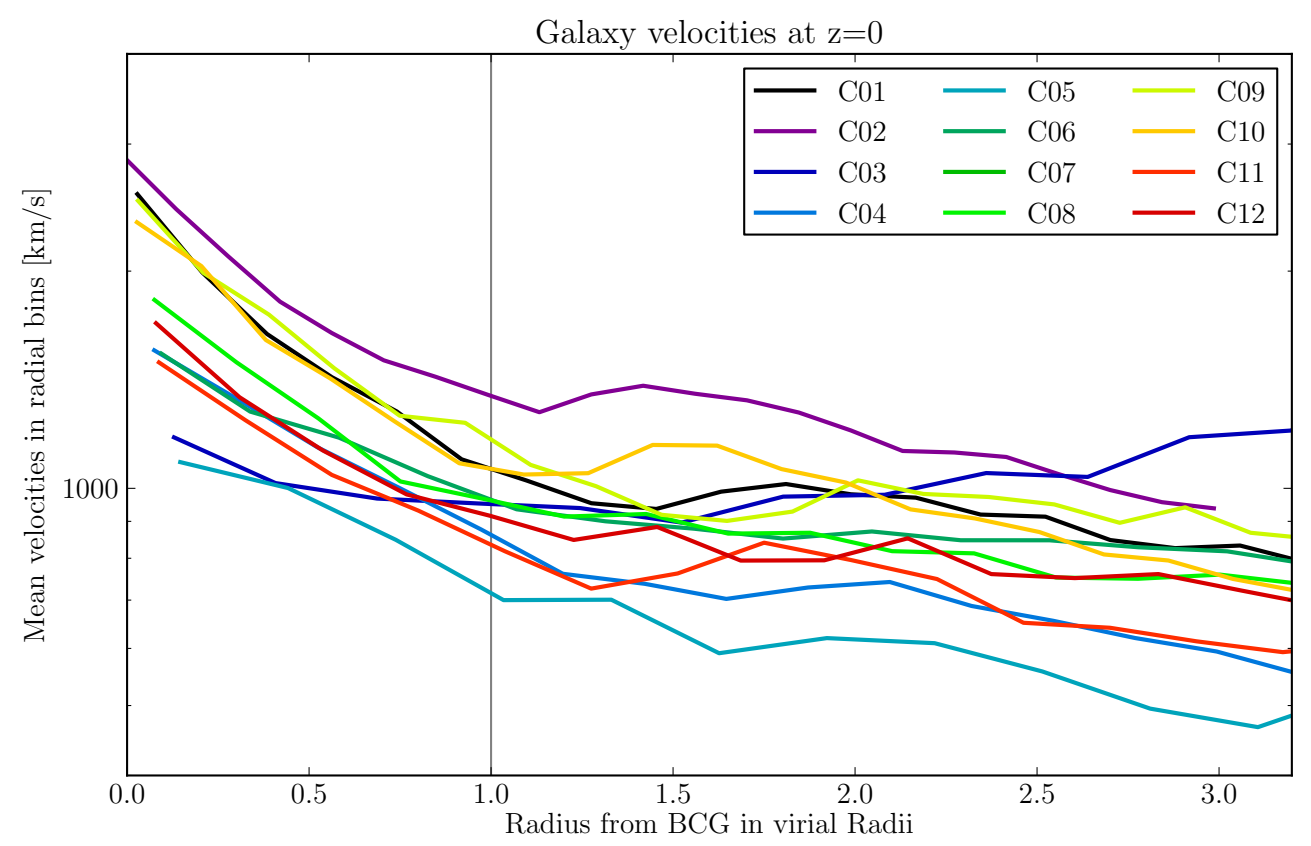

Fig. 6. Radially binned mean velocities.

\section{1. $\mathrm{C01}$}

The source $\mathrm{C} 01$ has a major subcluster merger event at $z \sim 0.7$ and another smaller merger at $z \sim 0.3$. In the graphical Appendix 7, we show the projected metallicities, DM density, temperature, and baroclinic vorticity (a quantity proportional to $\nabla p \times \nabla \rho$ that displays shock-induced turbulence, see Fig. A.3a) at $z=0$. In temperature and vorticity, the shock structures are visible. The elongated DM profile is another indicator of past mergers. $\mathrm{C} 01$ is a virializing, cool-core cluster with a peak temperature of $\sim 2 \times 10^{8} \mathrm{~K}$ at a cluster-centric radius $r \sim 0.2 \mathrm{Mpc}$, and a core temperature of $\sim 1.5 \times 10^{7} \mathrm{~K}$. In Figs. 12 and 9 (please note the different scales), we plot the radial profile of the metallicities due to galactic winds and disk ram-pressure stripping. Because $\mathrm{C} 01$ is a massive cluster, winds are effectively suppressed toward the center, and the level of RPS metal contribution is comparatively high. Interestingly, the sum of both contributions is almost constant out to the virial radius with values in the range $Z \sim 4-6 \times 10^{-3}$, except for the cool core where the metal mass-fraction obviously drops significantly below $1 / 3 Z_{\odot}$.

In Fig. 7 we plot the evolution of metallicities within the inner comoving $1 \mathrm{Mpc} / \mathrm{h}$. The lowest metallicity of the cool core is at the same level as the mean metallicity at a redshift $z>1$, which also coincides with the time of its formation. Furthermore, Fig. 7 shows a steady homogenization of metallicities toward $z=0.2$, but the variance (error bars) again increases at $z=0$, which is due to the onset of RPS.

The simulation of $\mathrm{C} 01$ with the wind model B ran into numerical problems with a cooling catastrophe already at redshift $z \sim 1.2$. Analysis shows that this wind prescription yields superolar metallicities for the major portion of the ICM already at such high redshifts. This suggests not only that the formation of cool cores is strongly correlated with the ICM metallicities, but also that the wind prescription from Hopkins et al. (2012) probably yields too high metallicities in the ICM. In Sects. 5.13 and 7 we discuss more arguments to support that claim, and we describe how we adapted the wind model for a more detailed analysis.

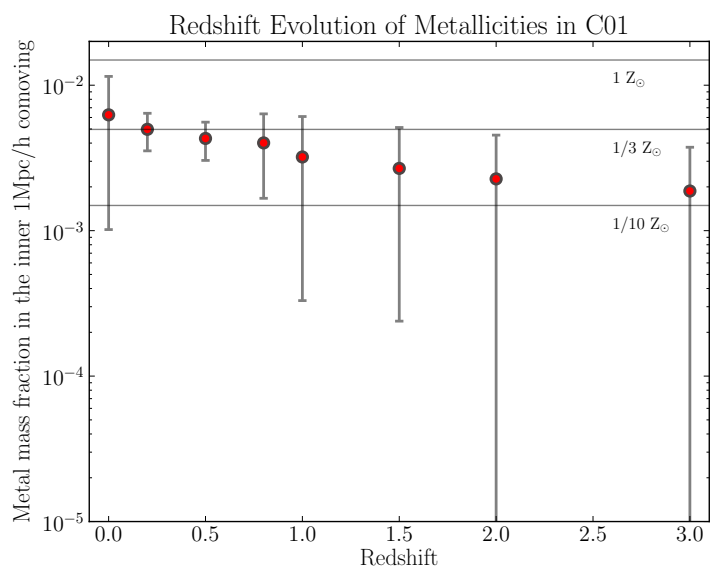

Fig. 7. Metallicity evolution C01 within a comoving $1 \mathrm{Mpc} / \mathrm{h}$ sphere around the density maximum. Plotted are mean values and the variance.

\section{2. $C 02$}

The source $\mathrm{C} 02$ is the most massive cluster in the sample (virial mass of $1.06 \times 10^{15} M_{\odot}$ ) and also has the highest peak galactic velocities in Fig. 6 . The cluster experiences a strong merger at $z \sim 0.3$ and there are two big groups (pre-merger) within $2 \mathrm{Mpc} / \mathrm{h}$ (one of which is cluster C03). The large DM halo in Fig. A.1b appears to be more elongated than it actually is because of a projection effect of an infalling subcluster along the $x$-direction. The relatively high level of SFR at $z=0$ in Fig. 3 does not show a significant correlation with the wind metallicity levels in the radial plot 12 toward the center. C02 also shows the steepest decline in wind metallicities within its virial radius, but it is among the highest wind metallicity objects of Fig. A.4b. The ram-pressure stripped metals in Fig. 9 are as expected amongst the absolute highest values in the sample. In Fig. A.3b we show the baroclinic vorticity, which is dominated by the pre-merger shocks of the infalling cluster $\mathrm{C} 03$ (coming from the lower right), but there is a considerable level of baroclinity also around the center, which traces the previous merger at $z \sim 0.3$. This event obviously was not able to disrupt the cool 
core, which we also find in $\mathrm{C} 02$, which likewise shows the significant drop in metallicity within the central $\sim 150 \mathrm{kpc}$.

\section{3. $\mathrm{C03}$}

Cluster C03 is not just a different final cut of the same volume as $\mathrm{C} 02$, but the whole data analysis presented in Sect. 2 was made individually for a shifted $20 \mathrm{Mpc} / \mathrm{h}$ side-length volume of the same $80 \mathrm{Mpc} / \mathrm{h}$ box. With this, we are able to test resolution effects of the nested grid approach.

The source $\mathrm{C} 03$ is a configuration centered on an infalling smaller subcluster of C02, see Fig. A.1c. The strong hydrodynamical shocks caused by the ongoing approach are seen in temperature and baroclinic vorticity (Figs. A.2 and A.3). The projected metal mass-fraction coming from galactic winds in Figs. A.4c shows an s-like shape that is not seen as clearly in b. This is mostly a projection effect, however. This special feature arises from the second infalling subcluster, which had a recent close fly-by between $\mathrm{C} 02$ and $\mathrm{C} 03$ with a trajectory spiraling toward the main cluster core. Because $\mathrm{C} 03$ is not an actual isolated galaxy cluster, this untypical behavior extends to the radial SFR, the radial wind metallicities plots, and the radial mean velocities (Figs. 5, 12, and 6). While all virialized or semivirialized objects in Fig. A.6 show ring-like structures in wind metals in the core cluster region, $\mathrm{C} 03$ as one of the untypical clusters presents a large cloud of dragged-along high-metallicity gas. All the relaxed systems show wind suppression in the high-density (and thus high-pressure) region of the cluster centers, as expected.

\subsection{C04}

The cluster $\mathrm{C} 04$ shows a major-merger event at $z \sim 0.6$ and ongoing wafting of the central halo for the rest of the simulation. This cluster has a relatively low mass, showing also one of the smallest velocity dispersions in Fig. 6. The contribution to the metal masses by disk ram-pressure stripping is generally low for $\mathrm{C} 04$, which is seen both in the radial metallicity profile in Fig. 9 and in the projected metal mass-fraction in Fig. A.5d. The RPS metals show much structure and a strong peak in the radial plot, which is due to recent stripping, especially in the very center of the cluster, while the overall (total and relative) contribution to the metallicity by ram-pressure stripping is significantly smaller than for cluster C01, for example. Especially in the zoomed-core total metal (wind + RPS) projection plots A.6, this cluster presents itself interestingly inhomogeneous with a high-metallicity region in the top left corner that is caused by two infalling groups that are already very close to the actual cluster at $z=0$, as seen in the dark matter projection A.1d. C04 shows a slightly cool core that is dislocated from the DM density peak by $\sim 220 \mathrm{kpc}$, where the temperature also peaks at $\sim 8 \times 10^{7} \mathrm{~K}$.

In Fig. 8 we plot the evolution of the central metallicity levels with time. The metallicity of $\mathrm{C} 04$ drops at about $z=1.5$ because of filament infall of poorly enriched gas. Figure 8 also shows an increased level of variance (i.e., inhomogeneity) at $z=0$ due to localized RPS peaks.

\section{5. $C 05$}

The cluster $\mathrm{C} 05$ has the lowest mass in our sample. It experiences a triple merger at $z \sim 0.3-0.2$ and relaxes to become semivirialized by $z=0$. There is a central cool core where the temperature drops from $\sim 3 \times 10^{7} \mathrm{~K}$ to $\sim 8 \times 10^{6} \mathrm{~K}$. The metallicity maps in Fig. A.4e show several extraordinarily

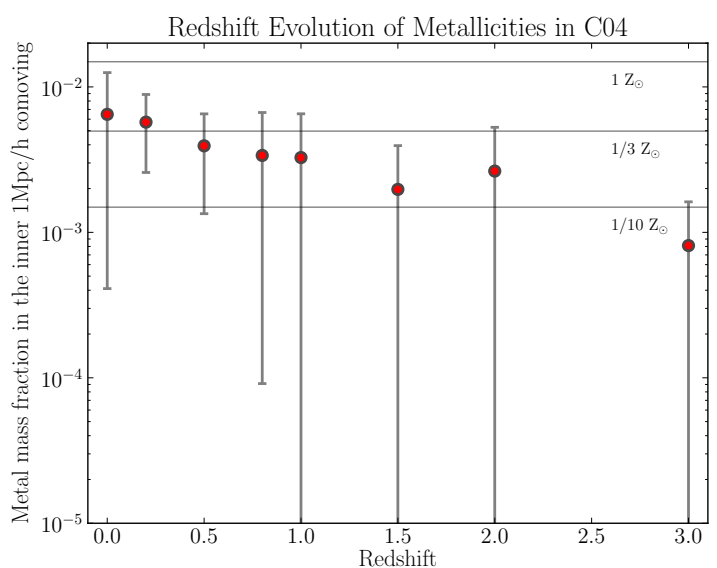

Fig. 8. Metallicity evolution of C04 within a comoving $1 \mathrm{Mpc} / \mathrm{h}$ sphere around the density maximum. Plotted are mean values and the variance.

high metallicity clumps within a rather low metallicity cluster environment.

The overall modest metallicity values are probably related to the lowest integrated SFRs in the sample (see Fig. 3).

\section{6. $\mathrm{Co6}$}

The cluster C06 shows a merger event at $z \sim 0.2$ and ongoing wafting thereafter until $z=0$. Nevertheless, there is a slight but extended cool core with a temperature contrast of approximately 0.4 within $250 \mathrm{kpc}$. Although $\mathrm{C} 06$ has the highest integrated SFR, the level of metal mass-fraction contribution from galactic winds is medium for most of the plotted volume in Fig. 12. There is a striking drop of metallicity toward the unvirialized cluster core, whose direction strongly correlates with that of the elongated DM halo, as seen in Figs. A.4f and A.1f. Figure A.4f shows that there are several high-metallicity regions outside the actual cluster that originate from infalling galaxies with recent high SFR episodes, while the very core remains depleted of wind metals. The $20 \mathrm{Mpc} / \mathrm{h}$ volume in whose center C06 resides is generally excessively busy and dense with a total matter density of $\Omega_{\mathrm{c}}=0.95$, which provides a good indication of the shape of the volume-averaged SFR history. The mass of C06 within $r_{200}$ of $1.5 \times 10^{14} M_{\odot}$ is similar to that of $\mathrm{C} 11$ and $\mathrm{C} 04$, which also manifests itself in very similar and rather low velocity dispersions (see Fig. 6). This means that disk rampressure stripping in C06 is much less significant than in highmass clusters. But we found a high-metallicity RPS peak close to the center, which is caused by recent stripping events. The radial wind-metallicity profile of this galaxy cluster is untypical, which shows the unique central depletion of wind metals that is also illustrated in the projected total metal mass-fraction in Fig. A.6f. This extended region of low metallicities is caused by the $z \sim 0.2$ merger of two early formed cool cores that have a metallicity level of high redshift, as discussed for previous clusters.

\section{7. $C 07$}

The configuration $\mathrm{C} 07$ is a pre-merger case of several subclusters at a distance of $\sim 1.5 \mathrm{Mpc} / \mathrm{h}$, evident in the baroclinic vorticity plot with a butterfly-like shock structure. Although the measured velocities of the galaxies in $\mathrm{C} 07$ are similar to relaxed systems of similar total mass, no extended static ICM halo has developed yet, and the contribution to metals by disk ram-pressure stripping is smaller than for the virialized cases, 
Radial profiles of RPS metallicities at $\mathrm{z}=0.0$

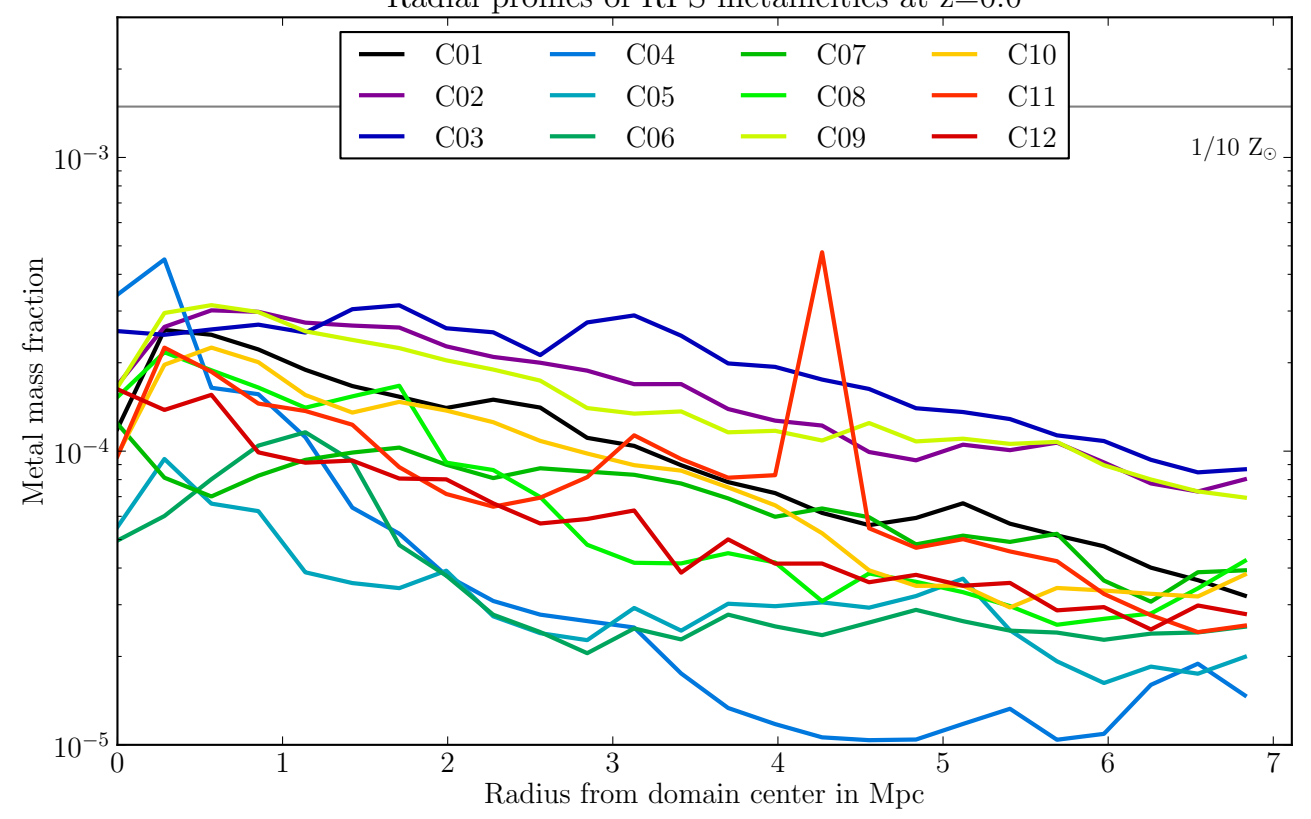

Fig. 9. Radial profiles of metal mass-fractions from RPS.

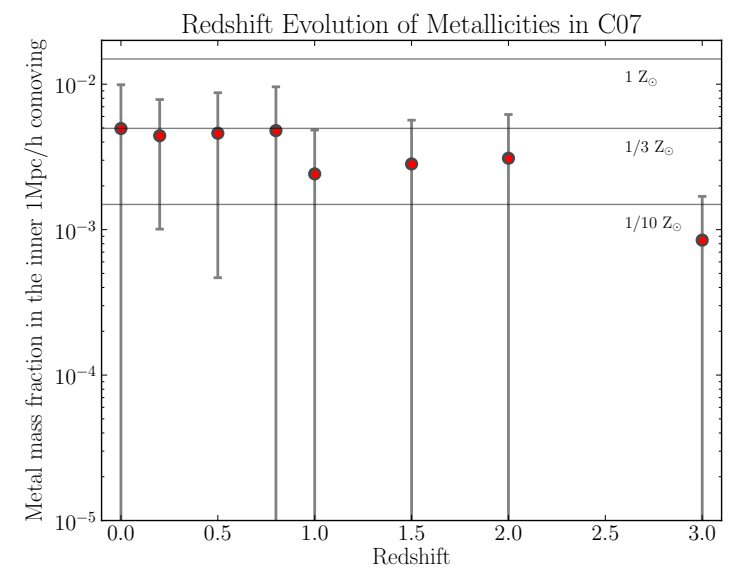

Fig. 10. Metallicity evolution of $\mathrm{C} 07$ within a comoving $1 \mathrm{Mpc} / \mathrm{h}$ sphere around the density maximum. Plotted are mean values and the variance.

while we find much structure in the RPS metallicity projection in Fig. A.5g. This suggests that the stripping events took place at low redshift. Because configuration C07 is not an actual galaxy cluster, a number of cool cores coincide with the DM peaks with temperature contrasts of $0.5-0.3$. The redshift evolution of metallicity in Fig. 10 shows a peculiar peak at $z=0.8$ that is similar to the final mean metallicity of $1 / 3 Z_{\odot}$ at $z=0.0$.

The DM structure in Fig. A.1g in the lower left corner seems close in the $x$-projection, but is at a distance of about $6 \mathrm{Mpc} / \mathrm{h}$.

\section{8. $C 08$}

Cluster C08 shows a major-merger event at $z \sim 1$ and after this, smooth and steady mass accretion. DM density, gas density, and pressure peak at the very center of the cluster, which also hosts a steep cool core. The baroclinity values are moderate, as expected for clusters with no recent significant merger events. In Fig. 12 the radial wind metal mass-fraction profile is plotted, showing as in the other cool-core cases a significant drop of metallicity at the very center. The peak at distance $6.6 \mathrm{Mpc}$ is caused by a combination of a recent starburst and an already very rarefied ICM in these outer parts of the cluster surroundings. Although the radial profile suggests a typical metallicity profile attributable to galactic winds, the projection plot in Fig. A.4h reveals an extended high-metallicity cloud in the upper center of the $x$-projection. Furthermore, it has a very localized metallicity peak caused by galactic winds within the inner Mpc of the cluster (not a projection effect), a peculiarity that is not seen in any other of the 12 configurations. This supersolar central metallicity peak is seen even more clearly in the zoomed-core total metallicity projection A.6h.

\section{9. $C 09$}

The cluster C09 shows major-merger events at $z \sim 1$ and $z \sim$ 0.7 and is virialized at redshift zero. For this object the radial trend in SFR (Fig. 5) is among the steepest within the sample. Furthermore, it has a very strong cool core where the temperature drops almost an order of magnitude in the inner $200 \mathrm{kpc}$ and as in the other cool-core cases on this length scale we also see a drop in metallicities. Because of its early formation as a galaxy cluster, it has one of the strongest contributions from RPS to the metallicities, but also a drop in this quantity toward the cool core (Fig. 9).

\subsection{0. $C 10$}

The DM plot in Fig. A.1j shows cluster C10 as a seemingly relaxed system, but the very high baroclinic vorticity values witness the very strong merger event at $z \sim 2$ with ongoing wafting until $z=0$. This very energetic event was obviously unable to prohibit the formation of a cool core, however. There is a peculiarity in temperature because of its kinetic state, as the temperature peak is more like an extended plateau between $0.2-1 \mathrm{Mpc}$. Figure A. 2 j reveals not only this large high-temperature region, but also the infall of a lower-temperature object from the left with a distinct bow shock in front of it. In Fig. 11 we plot the metallicity evolution in the inner comoving $\mathrm{Mpc} / \mathrm{h}$. This cluster 


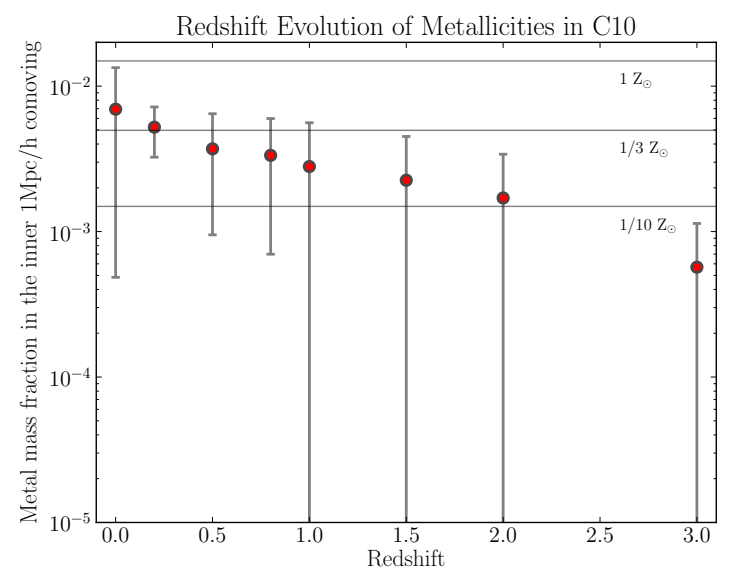

Fig. 11. Metallicity evolution of $\mathrm{C} 10$ within a comoving $1 \mathrm{Mpc} / \mathrm{h}$ sphere around the density maximum. Plotted are mean values and the variance.

also shows a steady homogenization of metallicities until the data point at $z=0.2$ and an increased variance at $z=0$.

\subsection{C11}

The cool-core cluster $\mathrm{C} 11$ also experiences a minor merger between $z \sim 0.2-0.1$. With its virial mass of $2.11 \times 10^{14} M_{\odot}, \mathrm{C} 11$ belongs to the lower mass clusters, which is also visible in the galaxy velocities in Fig. 6. However, its metallicity values within the virial radius are the highest in the sample, although they show the typical central drop in the cool core. The total metallicity projection in Fig. A.6k is remarkably symmetric for this virialized cluster. This may be also related with the generally very low SFR at $z=0$, seen both in the SFR history plot in Fig. 3 and in the radial SFR trend in Fig. 5, which suggests that the enrichment has occurred at an earlier epoch and the mixing within the ICM has effectively homogenized the metals.

\subsection{2. $C 12$}

The structure in C12 shows an ongoing multiple merger and is hence a very unvirialized object that also shows the most untypical radial SFR profile in Fig. 5, as well as a peculiar radial wind metallicity profile in Fig. 12. That the multiple merger is still in a very early stage explains why the vorticity is rather localized and the temperature peak is both high and comparatively extended, Figs. A.31 and A.21. There are two pre-merger cool cores dislocated from the shock-heated temperature maximum.

\subsection{Comparison of wind models}

As mentioned in Sect. 5.1, cluster C01 was simulated with the three different wind models we described in Sect. 3.1. Model B has very high efficiency values (Eq. (5)) for low-mass galaxies, yielding outflows of up to several thousand $M_{\odot} / \mathrm{yr}$ for starburst episodes. In combination with the assumption of solar metallicity of these winds, first simulations (without cooling) also showed ICM metallicities of almost constant value $Z_{\odot}$ at a redshift of about $z \sim 2$. Observations of ICM metallicities and galactic winds suggest that these values are hardly physical. Furthermore, the simulation of $\mathrm{C} 01$ with wind model B encountered numerical problems with a cooling catastrophe. In Fig. 14 we plot the radial profiles of the metal mass fraction of $\mathrm{C} 01$ from winds and the total for the three models. Here we ran model B with the efficiency factor from model A, see Eq. (3), from now on denoted as model B'.

Clearly, there are not only severe differences in the overall metallicity levels, but the shapes of the curves also differ significantly. The diversities are striking when the central projected metallicities are plotted in individual color scaling, see Fig. 13. For this plot, the actual scales are not relevant because we only wished to compare relative differences. If the models were reproducible by simply scaling the levels of metallicities, these projections should look very much alike, which they do not. One reason is of course the fractionally different contribution by ram-pressure stripping - the less efficient the wind model, the more small-scale structures are visible - but also when regarding the wind contribution alone (not plotted), the projections show a few common but also distinct features. As briefly reported in the cluster description, the cool-core metallicities roughly agree with the central mean metallicities of their formation times. The simulations with wind models A and B' have much lower metallicities, and the cool core forms much later and is shallower as well. The wind metallicity profiles of these two runs definitely show no drop in wind metals toward the center. However, the total metallicity shows a slight dip because the contribution from RPS metals in these two cases is larger in fraction. This suggests that after the cool core has formed, there is virtually no way to enrich it more by any process that we modeled in the setup. We discuss this peculiar feature in Sect. 6.3.

Without introducing any additional parameters to reproduce observed ICM metallicities, clearly only model $\mathrm{C}$ reaches values similar to those found in studies by Tamura et al. (2001), Durret et al. (2005), and Simionescu et al. (2009), for example. Without question, one could additionally design the two wind prescriptions from Hopkins et al. (2012) and Veilleux et al. (2005) specifically to achieve higher metallicities. But at this point we were more interested in the robustness of these two wind models that originate in galaxy-scale simulations without specific modifications. There obviously is a wind model with an efficiency between those of B and B' that could reproduce expected ICM metallicities. We will study this in a future paper.

\section{Comparison with observations}

\subsection{Observations of ICM metallicities}

Since the first X-ray observations of galaxy clusters, we know that the ICM is enriched by heavy elements. Several mechanisms have been proposed to explain how these metals were ejected into the ICM. Understanding whether the enrichment occurred before or after the cluster formation is also crucial to constrain the importance of each mechanism. Galactic winds, for example, are thought to be more important at high redshift because the star formation peaked around redshift $z \sim 2-3$ (Hopkins 2004), while disk ram-pressure stripping plays a major role in the regions of high density. Recently, Werner et al. (2013) analyzed a set of 84 Suzaku pointings of the Perseus cluster and derived the metallicity profile in eight different directions up to the virial radius. They found a "remarkably uniform iron abundance, as a function of radius", which suggests that the enrichment occurred before and during the formation of the cluster. In addition to the metallicity peak associated with the BCG, these data agree very well with the qualitative radial trend we found in most profiles for relaxed clusters within our sample. As in Werner et al. (2013), previous studies by Matsushita (2011), Urban et al. (2011), and Leccardi \& Molendi (2008), for instance, also showed either flat or slightly radially increasing 
Radial profile of wind metallicities at $\mathrm{z}=0.0$

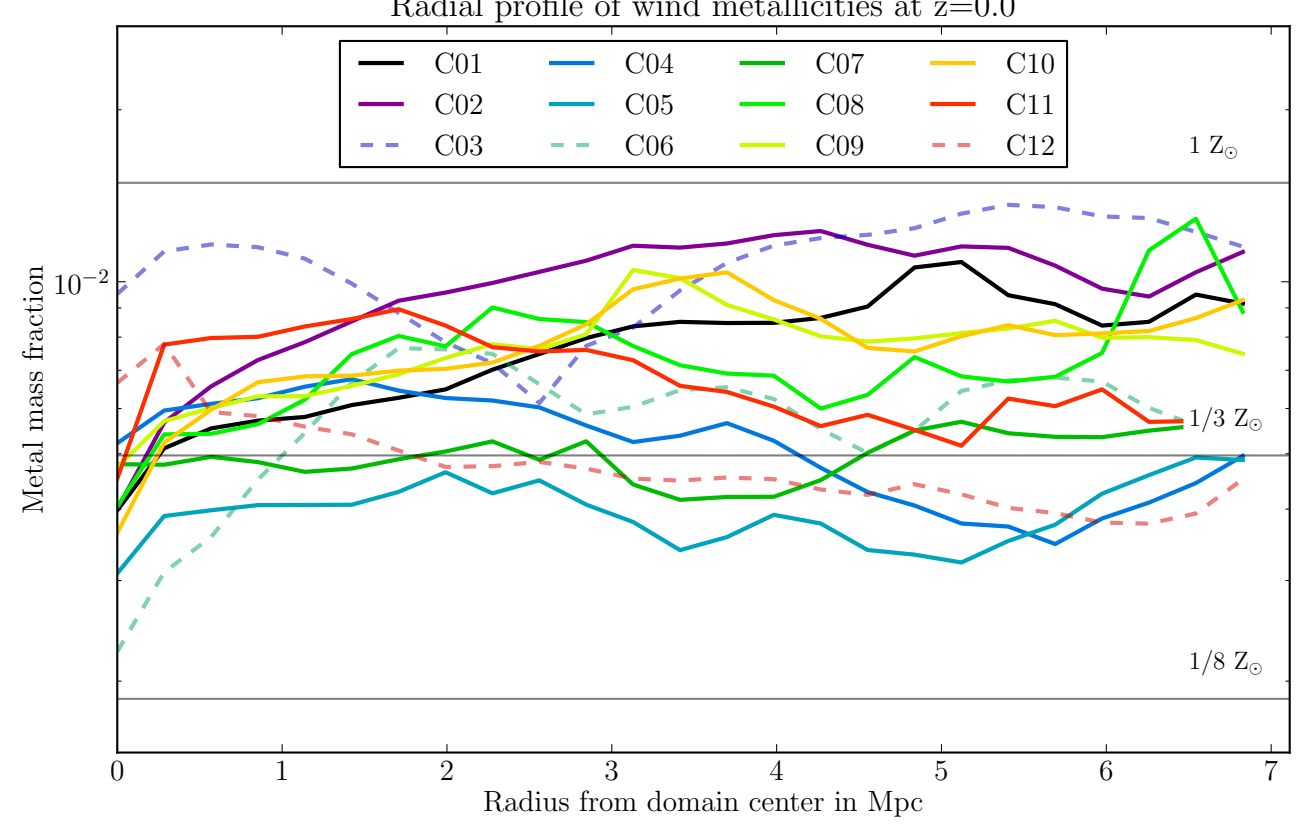

Fig. 12. Radial profiles of metal mass fractions from galactic winds. The dotted cases are classified as untypical because of their kinetic states.

metallicities in ranges typically between $1 / 10-1 Z_{\odot}$. However, while observational high-temperature metal data mainly refer to Fe measurements, the total metallicities within this work can be considered to be $\mathrm{O}$ dominated. Therefore a complete quantitative comparison will only be possible with single-element tracking, which is currently being implemented into the presented simulation setup and which will be introduced in future investigations. While there are only few observational single-element studies because of the stringent flux limitations, analyses by Tamura et al. (2004) and Matsushita et al. (2007) also found comparatively flat trends in $\mathrm{O}$. Another definite limitation in comparing the radial plot of Fig. 12 with the observational profiles is that in the simulation data we did not separate X-ray detectable ICM and gas that does not significantly contribute to the X-ray flux (which is proportional to $\rho^{2} \sqrt{T}$ ).

Because mixing within the ICM homogenizes the distribution of all metal species, the degree of structure seen in the metallicity is a promising tracer for the enrichment history. Radial profiles fail in detecting the local inhomogeneities of the metals, and detailed 2D metal maps are thus required. Metallicity maps, in fact, are the perfect tools for studying the efficiency of the different processes. Although we know that the metallicity distribution in galaxy clusters shows significant inhomogeneity even in relaxed clusters (e.g., Lovisari et al. 2011), we expect a more clumpy pattern for disturbed systems. One clear example of this is the distribution of metals in the famous merging cluster A3667 (Lovisari et al. 2009). The metal peak was detected to be between the two main substructures and probably caused by galactic winds associated with the new star formation triggered by the merging activity. This is not the only example, and other works showed that there are maxima in the metallicity distribution that are not associated with the cluster center (e.g., Durret et al. 2005; Simionescu et al. 2009; de Plaa et al. 2010; Kirkpatrick et al. 2011). A direct comparison between observations and simulations is now required not only for single objects, but also from the statistical point of view for a full understanding of the ICM enrichment. While most of the work until know focused on the iron distribution, a complete view of the metal-enrichment processes can be only obtained by additionally studying the distribution of other elements (e.g., O, Si, and S), as done by Sanders et al. (2004) for the Perseus cluster. Clearly, this requires a large number of photons (i.e., very long observations) and some prediction from the simulations. In 2015 ASTRO-H (Takahashi 2012) will be launched. One of its science goals is determining the metal distribution in galaxy clusters.

\subsection{Comparing the models with observations of galactic winds}

In Fig. 15 we compare observational estimates of galactic winds with the model predictions of the prescriptions presented in Sect. 3.1. While the observational data are very sparse, model B seems to overestimate the galactic wind at a given SFR and baryonic mass for all the objects. More data are definitely needed, especially for lower-mass systems and high SFRs - the galaxies that are thought to contribute a major fraction of ICM metals. Because the gravitational efficiency in models A and B' is zero for objects with a baryonic masses higher than $10^{9.4} M_{\odot}$ and the sample is strongly biased on the high mass end, there are some objects with total wind suppression. The two data points from Trotter et al. (1998) and Strickland \& Heckman (2009) seem to agree best with the Hopkins et al. (2012) prescription without the gravitational efficiency parameter.

\subsection{Simulation results}

As already mentioned in the previous section, the overall level of metals in the ICM in the simulations with wind model $\mathrm{C}$ agree very well with observations (see Fig. 16). There is a distinct difference between our results and a number of measurements of central metallicities, however. Usually, observers find a peak in central metallicity, especially in cool-core clusters (see, e.g., David et al. 2001; Sanders et al. 2004; Sanders \& Fabian 2007). But there are also observations that show the opposite trend (Sanderson et al. 2009) at the very center of cool-core clusters, which provide a striking indication of a bimodality 
H. Höller et al.: The distribution of metals in the ICM
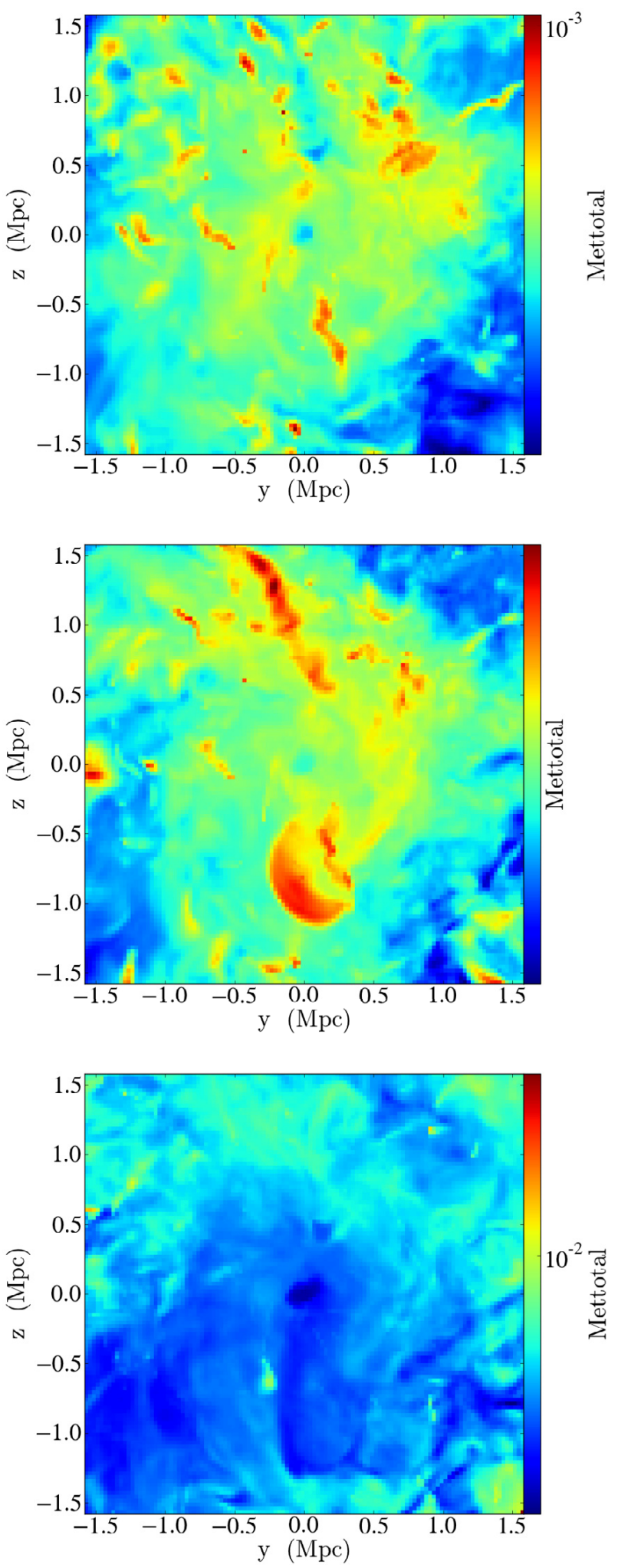

Fig. 13. Individually color-scaled (yt auto scaling) of X-ray fluxweighted metallicities with models A, B', and C in $x$-projections.

between CC and non-CC clusters. These observations were fitted with a single-temperature model for their spectra, which is considered less well suited than multitemperature profiles for coolcore clusters, see Buote (2000).

Our results showed an interestingly narrow distribution of core metallicities $\left(0.2-0.4 Z_{\odot}\right)$ when only virialized and
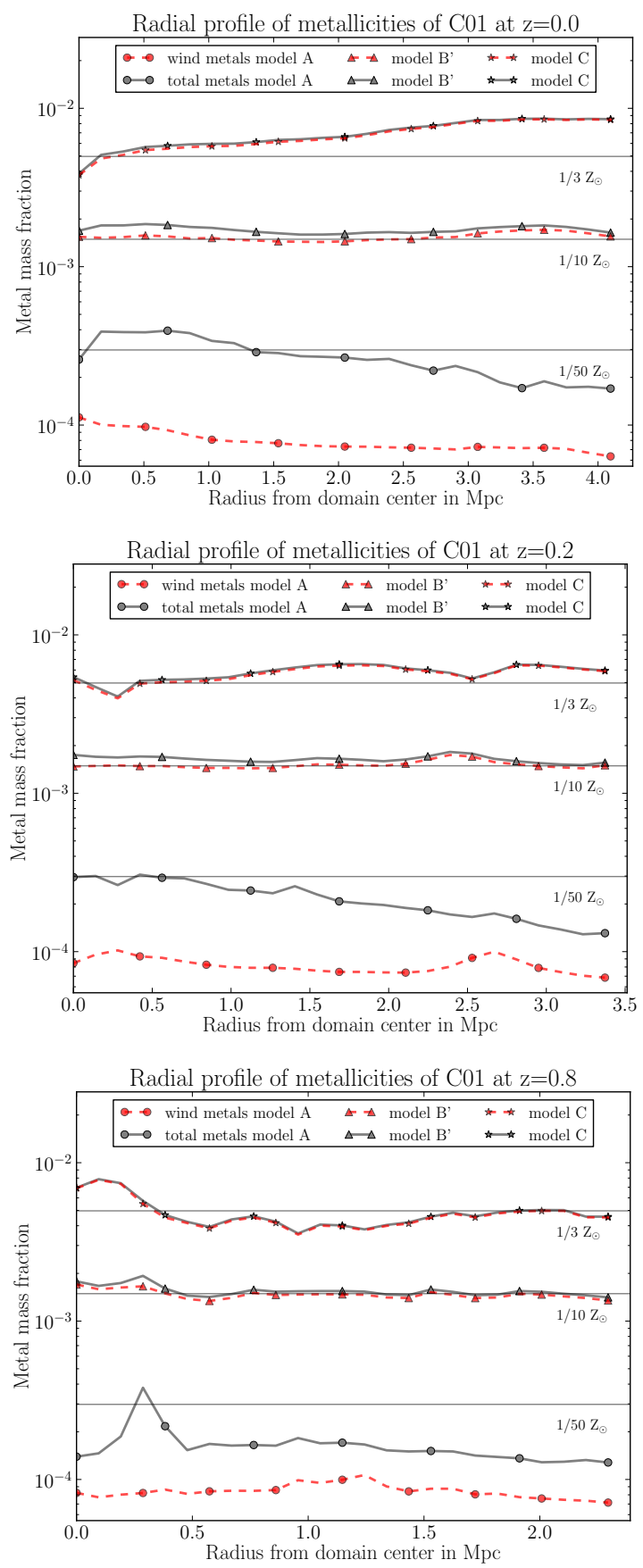

Fig. 14. Radial metallicity profile of $\mathrm{C} 01$ at showing the contributions from the three wind models at different redshifts.

semivirialized systems were considered. This result was also found observationally by de Grandi \& Molendi (2009). Our analysis showed striking evidence that the lack of mixing within and in the vicinity of the cool core causes the metal mass-fraction to freeze at the mean level of the time when the cool cores form (compare Figs. 7, 8, 10, and 11). While the region depleted of wind metals can be larger than the extent of the cool core itself, the depleted volume from ram-pressure-stripped metals is smaller in size and similar to or smaller than the cool core. This is probably best seen for cluster $\mathrm{C} 11$ by comparing Figs. A.5k and A.4k.

However, this finding should not be overinterpreted, because many numerical studies have shown a tendency to form cool cores in cluster simulations without introducing additional 
Galactic wind comparison observation/models

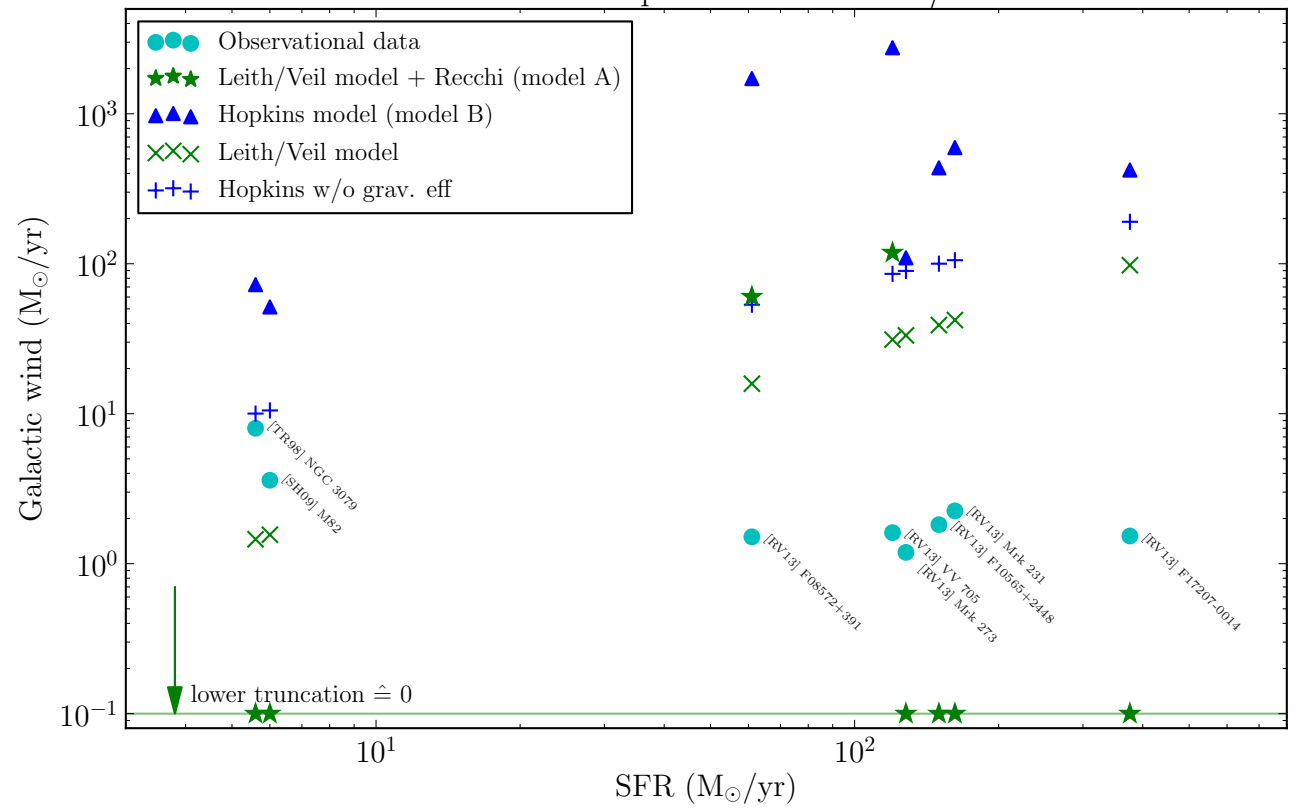

Fig. 15. Comparison of wind observations with models. Data on star formation rates and galactic winds taken from Rupke \& Veilleux (2013), Strickland \& Heckman (2009), Trotter et al. (1998), mass estimates taken from Dasyra et al. (2006) and $S_{0.5}$ fits for baryonic mass determination taken from Catinella et al. (2012).

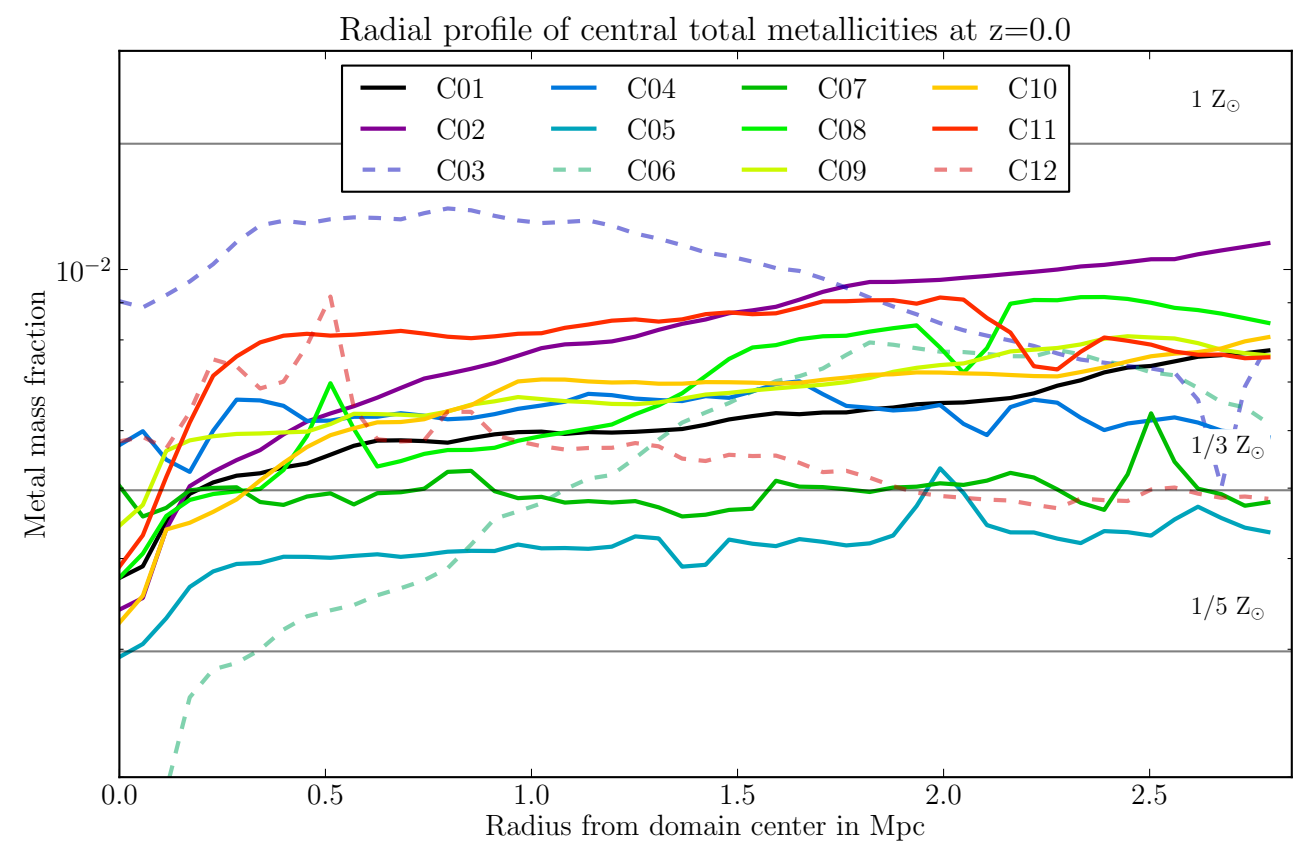

Fig. 16. Radial profiles of the total metal mass-fractions. The dotted cases are classified as untypical because of their kinetic states.

heating processes, such as AGN feedback. In this sense, the cool cores presented in this study are probably artificially deep and generally more prone to form than in models with additional heating prescriptions. It is remarkable, however, how well the core values agree with the mean metallicity values at the redshifts when they form (usually $z \sim 1.5-0.8$ ). There is no currently modeled physical process that would explain an increase in metallicities toward the cool cores. The metal mass-fraction would not show any gradient if mixing were completely perfect; the less efficient the mixing, the lower the metallicity in the core, one would assume. An increase in core metal massfraction can only be explained if the metals are produced in the center. With the current semi-analytical model GALACTICUS, we do not see significant SFR in the BCGs that would yield an appropriate amount of SN-driven metal outflows within the model presented in Sect. 3.1. The early formation of a dense, high-pressure core suppresses galactic winds in this region. The length scale over which the ICM metallicity drops in Fig. 16 is in the order of $200 \mathrm{kpc}$. This length scale is clearly associated with the BCG, a giant elliptical galaxy that (at the current setup status) is treated equally with all other galaxies. However, the characteristic length scales of a cD galaxy are significantly larger than the central resolution of the hydrodynamical simulation $(20 \mathrm{kpc} / \mathrm{h})$, and the gas residing in this object should be 
considered a part of the ICM, while in our model it is not transported into the ICM reservoir because of the mentioned wind suppression. While this weakness of our simulation setup will be removed in future revisions, we found this interesting mechanism of metallicity freezing in the cluster cores, which present themselves almost unmixed with the surrounding ICM. This result also supports the hypothesis of (Boehringer et al. 2004), who analyzed iron-to-silicon ratios in CC clusters. They claimed that "the central excess seen in cooling core clusters is most probably due to enrichment by SN type Ia in the cD galaxies in the recent past, during which the central region of the cluster was not disturbed". While we see no indications that turbulent mixing is only suppressed recently, but rather from the time of CC formation on, we will study this phenomenon in the future in detail and also apply single-element tracking in the feedback processes.

Moreover, although the inner $2.5 \mathrm{Mpc}$ as plotted in Fig. 16 accounts for less than $1 / 1000$ of the total simulated volume, there might be a radial bias toward higher metallicities in more massive systems because the drag of gaseous material other than DM is only taken from the $28 \mathrm{Mpc}^{3}$ box, and the inflow over the boxes boundaries is definitely underestimated. Comparisons of radial density profiles have shown, however, that this effect is noticeable only for the highest-mass objects, such as $\mathrm{C} 01$ and C02 in this sample.

In Sect. 6.1 we showed that to study the kinetic state of a galaxy cluster and the efficiency of the diverse enrichment processes, it is desirable to compare 2D metallicity maps with simulations. The richness of structures in metal mass-fraction is not shown to its full extent because we used common color maps (see Fig. A.6) in a rather diverse sample of objects. The X-ray flux-weighted metallicity projections nevertheless show a wide spectrum of metal distributions. Although we are aware of the small sample and the degeneracy of the projection direction, we wish to point out a few common but distinct features. This work should be considered as the basis for more systematic and more homogeneous choices of cluster samples for statistical analysis in conjunction with observational data.

Apart from in situ subgrid model simulations such as that by Tornatore et al. (2007), clusters in all kinetical states show structures and inhomogeneities of metallicities on galactic scales. Metallicity peaks are directly associated with starburst episodes or ram-pressure stripping events of single galaxies modeled by the SAM.

- High-mass relaxed systems show efficient mixing of galactic wind metals within the shock-heated ICM volume. Clusters $\mathrm{C} 01, \mathrm{C} 09$, and $\mathrm{C} 11$ show a remarkably symmetric spatial metal distribution on large scales (Figs. A.6a,i, and k). However, this degree of homogeneity is to be understood as relative to the other investigated systems. The central region of $\mathrm{C} 01$ is plotted in Fig. 17 in a $(y, z)$-slice. It shows much small-scale inhomogeneities and structures - in this case using a slightly different color scale of $0.13-1.3 Z_{\odot}-$ that trace recent stripping events and wind mass-loss of single objects. There is a radial break in large-scale homogeneity at the edge of the shocked ICM; the infalling gas shows much higher metallicity contrasts (orders of magnitude). A strong metallicity depletion of the cool core is a another common feature of these clusters.

- The cluster C02 has undergone a major-merger event at $z \sim 0.2$, the cluster is not virialized. The metallicity projection suggests two planes of symmetry, along and perpendicular to the merger direction. Although not a direct observable, the RPS metals in C10 (Fig. A.5b) show much structure,

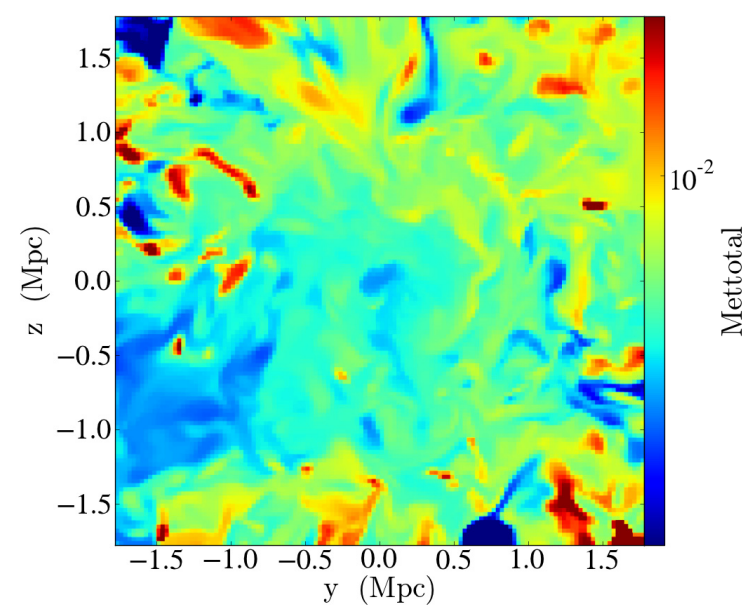

Fig. 17. Central $(y, z)$-slice of the total metal mass-fraction of cluster $\mathrm{C} 01$ at $z=0$ showing galactic-scale inhomogeneities within the ICM. The color range is equivalent to the interval $0.13-1.3 Z_{\odot}$.

an indication that the shock-induced turbulence has only recently started to efficiently mix the material.

- The lowest-mass clusters display also the lowest level of metal mass-fraction, while $\mathrm{C} 05$, a relaxed system, again shows a high degree of radial symmetry, while the premerger object $\mathrm{C} 07$ presents itself much more structured and inhomogeneous, see Fig. A.6g.

- The two pre-merger subclusters in C06 reveal unusually large volumes of metallicity depletion, while the projection in Fig. A.6f shows much structure and comparatively high metallicity values outside these regions.

- The multiple-merger system C12 displays an interesting bimodality where the upper left part in the $x-y$ projection is smoother than the inhomogeneous lower right subcluster(s). The elongated supersolar metallicity peak at the center coincides with a heavy shock from the ongoing merger.

- The low-metallicity, cool infalling structure in the left upper part of the metal mass-fraction projection of C10, Fig. A.6j, pushes a higher metallicity bow-shock region in front of it.

- As also seen in the radial profiles, all cool-core clusters show a decrease in metallicity at their centers. The spatial extent of these low metal mass-fraction regions is smaller in RPS metal contribution than in wind metals.

\section{Summary and outlook}

We presented a study on the temporal and spatial distribution of metals in the ICM within a heterogeneous sample of 12 cosmological simulations of galaxy clusters. We applied a novel simulation setup that combines a solid and comprehensive subgrid model by including the well-tested semi-analytical model GALACTICUS in grid-based hydrodynamical simulations. The strength of this setup is to robustly model the histories of a large number of objects (tens of thousands of galaxies) based on their merger histories within a high-resolution hydrodynamical treatment of the ICM, which facilitates studying the dynamics and chemical evolution.

The main results can be summarized as follows:

- The combination of the semi-analytical galaxy formation model GALACTICUS and a grid-based hydrodynamical cosmological simulation has proven reliable in reproducing a number of observables, such as the star formation histories, 
a radial trend in SFR within the galaxy cluster environment, and the overall level of metallicities in the ICM.

- The diverse galaxy cluster samples show common and distinct features in radial trends and 2D metallicity projections. Relaxed objects display efficiently mixed metal mass-fractions within the shock-heated regions. Kinetically peculiar clusters display significantly more structure and individual characteristics. 2D metallicity maps are more adequate for a detailed analysis of feedback process efficiencies and for tracing the kinetic state of galaxy clusters.

- Galactic winds dominate the overall contribution to ICM metals, while RPS adds at low redshifts and shows a significant radial trend. The radial course in total metal massfraction shows less spread at smaller radii, and cool-core clusters are metallicity depleted. The analysis showed that the mean metal mass-fraction values at the time of the formation of the CCs coincides with the final level within the central few hundred kpc. Turbulent mixing of ICM material within the CCs is heavily suppressed in our simulations.

- The comparison of wind models showed that without additional parameters and/or models, only wind model $\mathrm{C}$ can reproduce metallicity values similar to those seen in $\mathrm{X}$-ray observations. The formation time of the cool cores strongly depends on the ICM metallicities; depth and size of the metallicity dip in CCs is smaller the later they form.

- The main weakness of the simulation setup in the current status is that it neglects the ISM within the BCG, which is the most likely cause for the metal mass-fraction drop in the cluster cores. Results suggest that this material must be considered as part of the ICM in future studies.

The next step is the implementation of single-element tracking into our feedback models. This way, the comparison with observational data will be even more effective and reliable in analyzing the feedback process efficiencies as a function of space and time. We are furthermore working on an implementation of GALACTICUS in which ram-pressure stripping is computed from real relative velocities and external ICM densities. This next release will reproduce even more realistic SFRs within galaxy clusters and also model star formation quenching from disk rampressure stripping.

We will analyze the formation of cool cores when AGN feedback is included in the computations. Furthermore, the gas that is currently captured in the BCG by suppression of the transport process will be considered part of the ICM. In combination with the single-element tracking, we aim to be able to test the hypothesis of Boehringer et al. (2004), who claimed that the central metals originate more likely from supernovae type Ia in these central elliptical galaxies.

Acknowledgements. The authors acknowledge the UniInfrastrukturprogramm des BMWF Forschungsprojekt Konsortium Hochleistungsrechnen, the Forschungsplattform Scientific Computing at LFU Innsbruck and the doctoral school - Computational Interdisciplinary Modelling FWF DK-plus (W1227). The software used in this work was in part developed by the DOE NNSA-ASC OASCR Flash Center at the University of Chicago. We thank Matteo Bianconi, Asmus Böhm, Benjamin Bösch, and Sabine Schindler for many valuable discussions and their perspective from observational (X-ray) astronomy. We furthermore acknowledge the anonymous referee, who helped to substantially improve the paper.

\section{References}

Adelberger, K. L., Steidel, C. C., Shapley, A. E., \& Pettini, M. 2003, ApJ, 584, 45

Allen, S. W., \& Fabian, A. C. 1998, MNRAS, 297, L63
Arnaud, M., Rothenflug, R., Boulade, O., Vigroux, L., \& Vangioni-Flam, E. 1992, A\&A, 254, 49

Asplund, M., Grevesse, N., Sauval, A. J., \& Scott, P. 2009, ARA\&A, 47, 481

Behroozi, P. S., Wechsler, R. H., \& Wu, H.-Y. 2013a, ApJ, 762, 109

Behroozi, P. S., Wechsler, R. H., Wu, H.-Y., et al. 2013b, ApJ, 763, 18

Benson, A. J. 2010, Phys. Rep., 495, 33

Benson, A. J. 2012, New Astron., 17, 175

Benson, A. J., \& Bower, R. 2011, MNRAS, 410, 2653

Benson, A. J., Borgani, S., De Lucia, G., Boylan-Kolchin, M., \& Monaco, P. 2012, MNRAS, 419, 3590

Boehringer, H., Matsushita, K., Churazov, E., \& Finoguenov, A. 2004, in The Riddle of Cooling Flows in Galaxies and Clusters of galaxies, eds. T. Reiprich, J. Kempner, \& N. Soker, 189

Boylan-Kolchin, M., Ma, C.-P., \& Quataert, E. 2008, MNRAS, 383, 93

Bresolin, F., Kennicutt, R. C., \& Ryan-Weber, E. 2012, ApJ, 750, 122

Buote, D. A. 2000, MNRAS, 311, 176

Cassano, R., Ettori, S., Giacintucci, S., et al. 2010, ApJ, 721, L82

Catinella, B., Kauffmann, G., Schiminovich, D., et al. 2012, MNRAS, 420, 1959

Cole, S., Lacey, C. G., Baugh, C. M., \& Frenk, C. S. 2002, MNRAS, 319, 168

Dasyra, K. M., Tacconi, L. J., Davies, R. I., et al. 2006, ApJ, 638, 745

David, L. P., Nulsen, P. E. J., McNamara, B. R., et al. 2001, ApJ, 557, 546

de Grandi, S., \& Molendi, S. 2009, A\&A, 508, 565

de Plaa, J., Grange, Y. G., Kaastra, J. S., Werner, N., \& Simionescu, A. 2010,

X-ray Astronomy 2009; Present Status, Multi-Wavelength Approach and

Future Perspectives, AIP Conf. Proc., 1248, 289

De Young, D. S. 1978, ApJ, 223, 47

De Young, D. S. 1986, ApJ, 307, 62

Dressler, A. 1980, ApJ, 236, 351

Durret, F., Lima Neto, G. B., \& Forman, W. 2005, A\&A, 432, 809

Eisenstein, D. J., \& Hu, W. 1998, ApJ, 496, 605

Fabian, A. C. 1994, ARA\&A, 32, 277

Fabian, A. C. 2012, ARA\&A, 50, 455

Ferguson, A. M. N., Gallagher, J. S., \& Wyse, R. F. G. 1998, AJ, 116, 673

Font, A. S., Bower, R. G., McCarthy, I. G., et al. 2008, MNRAS, 389, 1619

Gerhard, O., Arnaboldi, M., Freeman, K. C., \& Okamura, S. 2002, ApJ, 580, L121

Gonzalez Delgado, R. M., Leitherer, C., Heckman, T., et al. 1998, ApJ, 495, 698

Gunn, J. E., Gott, J., \& Richard, I. 1972, ApJ, 176, 1

Hahn, O., \& Abel, T. 2011, MNRAS, 415, 2101

Heckman, T. M., Armus, L., \& Miley, G. K. 1990, ApJS, 74, 833

Höller, H. 2014, Ph.D. Thesis, Universit of Innsbruck, Institut for Astro- and Particle Physics

Hopkins, A. M. 2004, ApJ, 615, 209

Hopkins, P. F., Quataert, E., \& Murray, N. 2012, MNRAS, 421, 3522

Matsushita, K., Böhringer, H., Takahashi, I., \& Ikebe, Y., 2007, A\&A, 462, 953

Kapferer, W., Kronberger, T., Breitschwerdt, D., et al. 2009, A\&A, 504, 719

Kirkpatrick, C. C., McNamara, B. R., \& Cavagnolo, K. W. 2011, ApJ, 731, L23

Kistler, M. D., Yüksel, H., Beacom, J. F., Hopkins, A. M., \& Wyithe, J. S. B. 2009, ApJ, 705, L104

Knebe, A., Knollmann, S. R., Muldrew, S. I., et al. 2011 [arXiv: 1104. 0949]

Larson, D., Dunkley, J., Hinshaw, G., et al. 2011, ApJS, 192, 16

Leccardi, A., \& Molendi, S. 2008, A\&A, 487, 461

Leitherer, C., Schaerer, D., Goldader, J. D., et al. 1999, ApJS, 123, 3

Lewis, I., Balogh, M., De Propris, R., et al. 2002, MNRAS, 334, 673

Lodders, K. 2003, ApJ, 591, 1220

Lovisari, L., Kapferer, W., Schindler, S., \& Ferrari, C. 2009, A\&A, 508, 191

Lovisari, L., Schindler, S., \& Kapferer, W. 2011, A\&A, 528, A60

Markevitch, M., Ponman, T. J., Nulsen, P. E. J., et al. 2000, ApJ, 541, 542

Matsushita, K. 2011, A\&A, 527, A134

Maughan, B. J., Jones, C., Forman, W., \& Van Speybroeck, L. 2008, ApJS, 174, 117

Meurer, G. R., Heckman, T. M., Leitherer, C., et al. 1995, AJ, 110, 2665

Mitchell, R. J., Culhane, J. L., Davison, P. J. N., \& Ives, J. C. 1976, MNRAS, $175,29 \mathrm{P}$

Oemler, J. A. 1974, ApJ, 194, 1

Poole, G. B., Fardal, M. A., Babul, A., et al. 2006, MNRAS, 373, 881

Pranger, F., Böhm, A., Ferrari, C., et al. 2013, A\&A, 557, A62

Quilis, V., Moore, B., \& Bower, R. 2000, Science, 288, 1617

Recchi, S., \& Hensler, G. 2013, A\&A, 551, A41

Rodionov, S. A., \& Sotnikova, N. Y. 2005, Astron. Rep., 49, 470

Rupke, D. S. N., \& Veilleux, S. 2013, ApJ, 768, 75

Sanders, J. S., \& Fabian, A. C. 2007, MNRAS, 381, 1381

Sanders, J. S., Fabian, A. C., Allen, S. W., \& Schmidt, R. W. 2004, MNRAS, 349,952

Sanderson, A. J. R., O’Sullivan, E., \& Ponman, T. J. 2009, MNRAS, 395, 764

Sarazin, C. 1986, Rev. Mod. Phys., 58, 1

Scarano, S., \& Lépine, J. R. D. 2013, MNRAS, 428, 625 
H. Höller et al.: The distribution of metals in the ICM

Schaap, W. E., \& van de Weygaert, R. 2000, A\&A, 363, 29

Shaver, P. A., McGee, R. X., Newton, L. M., Danks, A. C., \& Pottasch, S. R. 1983, MNRAS, 204, 53

Simionescu, A., Werner, N., Böhringer, H., et al. 2009, A\&A, 493, 409

Springel, V. 2005, MNRAS, 364, 1105

Springel, V., \& Hernquist, L. 2003, MNRAS, 339, 312

Steidel, C. C., Erb, D. K., Shapley, A. E., et al. 2010, ApJ, 717, 289

Steinhauser, D., Haider, M., Kapferer, W., \& Schindler, S. 2012, A\&A, 544, A54

Strickland, D. K., \& Heckman, T. M. 2009, ApJ, 697, 2030

Su, M., Slatyer, T. R., \& Finkbeiner, D. P. 2010, ApJ, 724, 1044

Sutherland, R. S., \& Dopita, M. A. 1993, ApJS, 88, 253

Tamura, T., Kaastra, J. S., Peterson, J. R., et al. 2001, A\&A, 365, L87
Tamura, T., Kaastra, J. S., den Herder, J. W. A., Bleeker, J. A. M., \& Peterson., J. R. 2004, A\&A, 420, 135

Takahashi, E. 2012, in SPIE Conf. Ser., 8443, 3

Tinker, J., Kravtsov, A. V., Klypin, A., et al. 2008, ApJ, 688, 709

Tornatore, L., Borgani, S., Dolag, K., \& Matteucci, F. 2007, MNRAS, 382, 1050

Trotter, A. S., Greenhill, L. J., Moran, J. M., et al. 1998, ApJ, 495, 740

Urban, O., Werner, N., Simionescu, A., Allen, S. W., \& Böhringer, H. 2011, MNRAS, 414, 2101

Veilleux, S., Cecil, G., \& Bland-Hawthorn, J. 2005, ARA\&A, 43, 769

Vogt, N. P., Haynes, M. P., Giovanelli, R., \& Herter, T. 2004, AJ, 127, 3300

Werner, N., Urban, O., Simionescu, A., \& Allen, S. W. 2013, Nature, 502, 656

Wills, B. J., Netzer, H., \& Wills, D. 1985, ApJ, 288, 94

Zaritsky, D., Kennicutt, Jr., R. C., \& Huchra, J. P. 1994, ApJ, 420, 87 


\section{Appendix A}

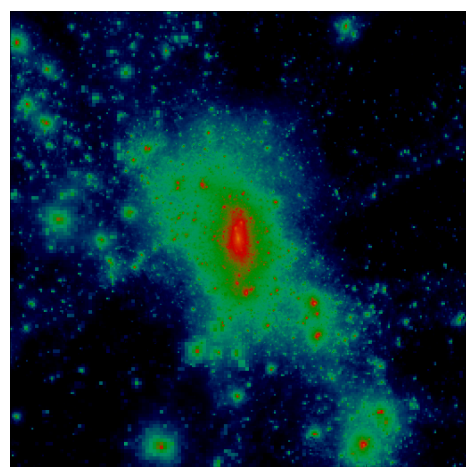

(a) $\mathrm{C} 01$

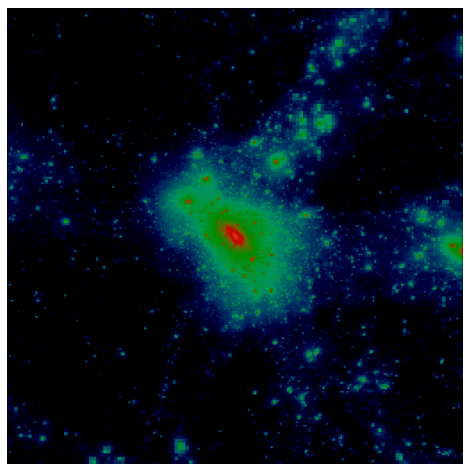

(d) $\mathrm{C} 04$

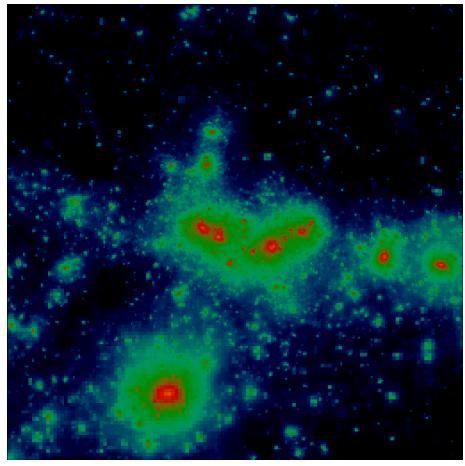

(g) $\mathrm{C} 07$

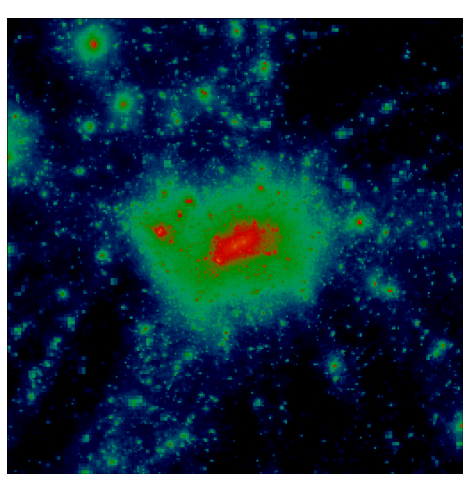

(j) $\mathrm{C} 10$

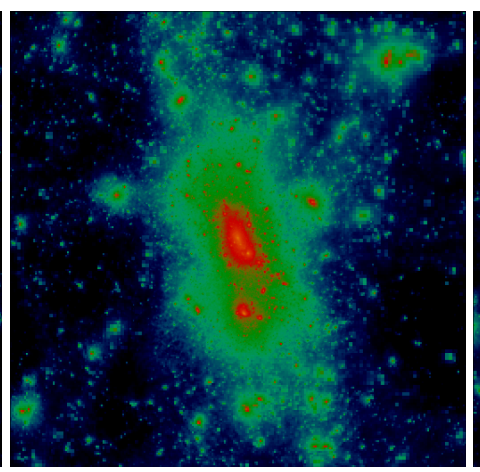

(b) $\mathrm{C} 02$

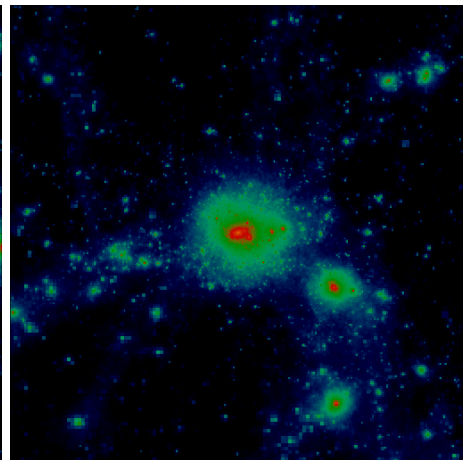

(e) $\mathrm{C} 05$

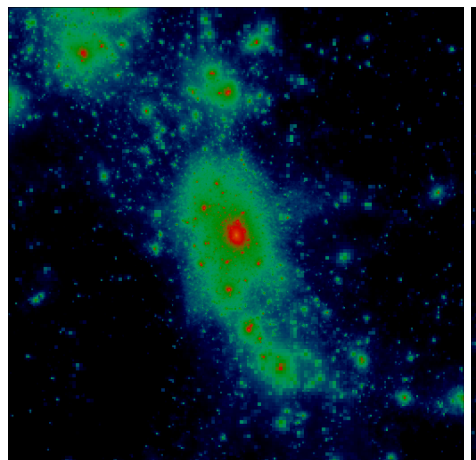

(h) $\mathrm{C} 08$

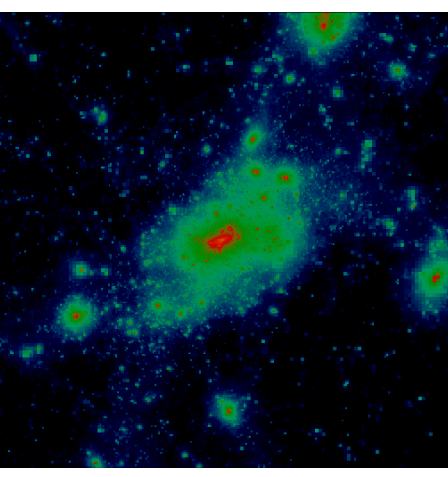

(k) $\mathrm{C} 11$

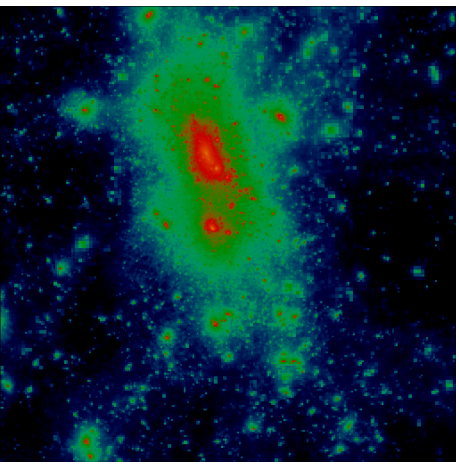

(c) $\mathrm{C} 03$

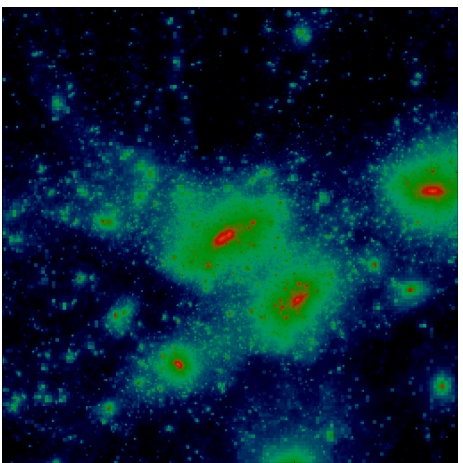

(f) $\mathrm{C} 06$

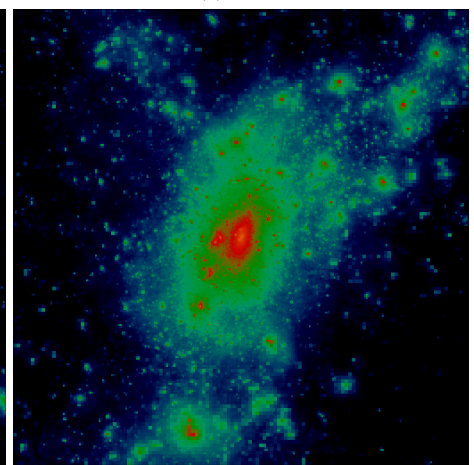

(i) $\mathrm{C} 09$

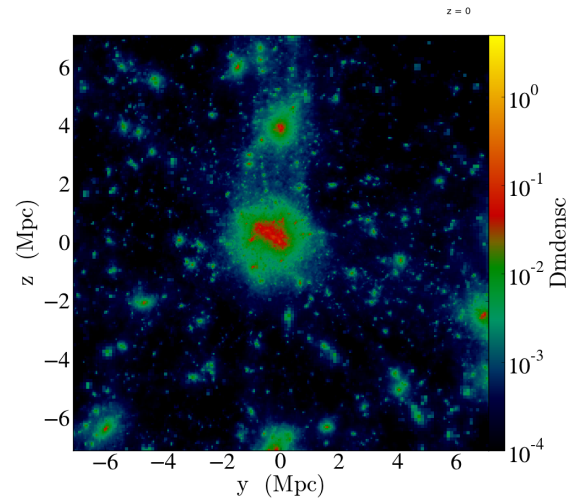

(1) $\mathrm{C} 12$

Fig. A.1. Dark matter density $x$-projections in $\mathrm{g} / \mathrm{cm}^{2}$ of all 12 clusters. All plots show the inner $5 \mathrm{Mpc} / \mathrm{h}$ and use the same color scheme, see Fig. 1. 
H. Höller et al.: The distribution of metals in the ICM

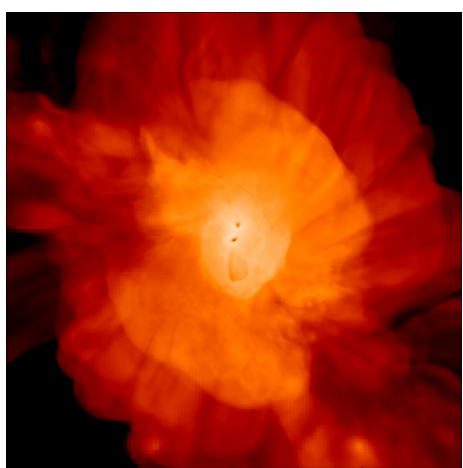

(a) $\mathrm{C} 01$

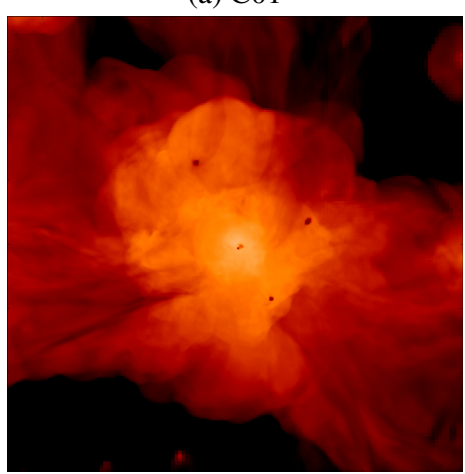

(d) $\mathrm{C} 04$

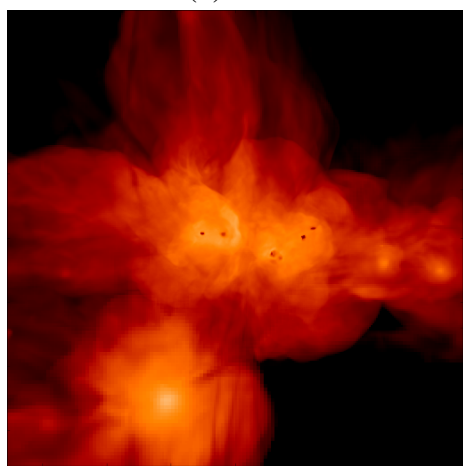

(g) $\mathrm{C} 07$

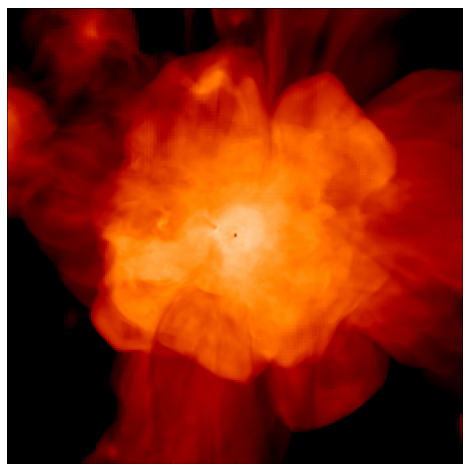

(j) $\mathrm{C} 10$

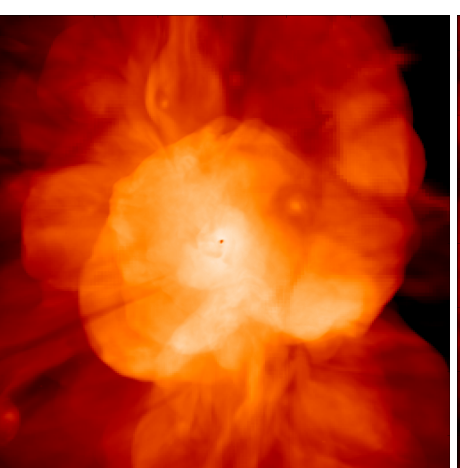

(b) $\mathrm{C} 02$

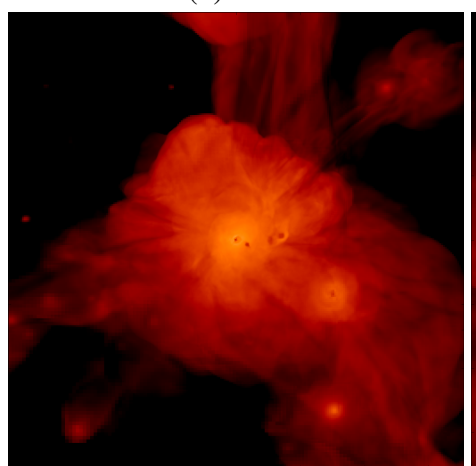

(e) $\mathrm{C} 05$

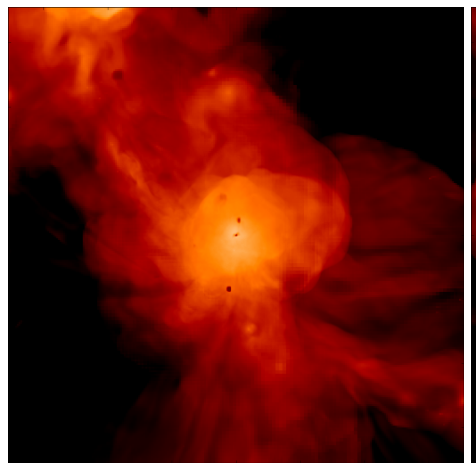

(h) $\mathrm{C} 08$

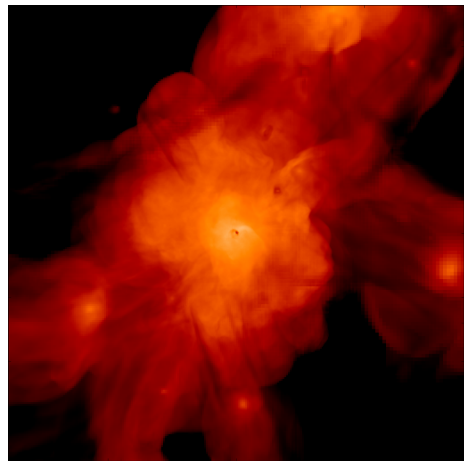

(k) $\mathrm{C} 11$

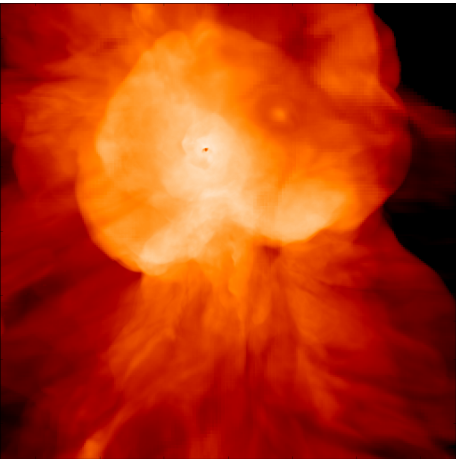

(c) $\mathrm{C} 03$

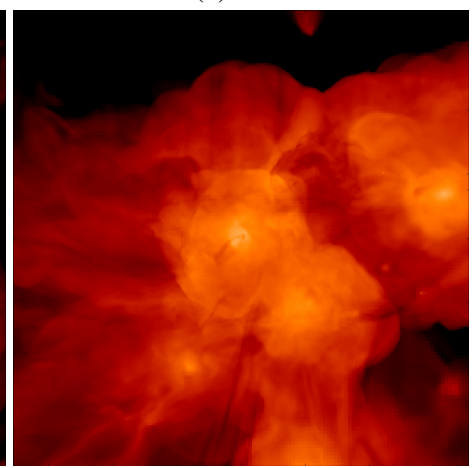

(f) $\mathrm{C} 06$

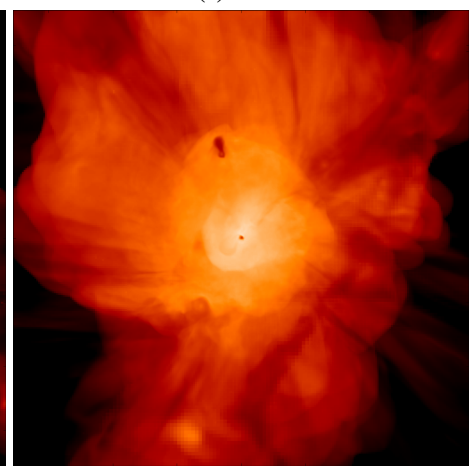

(i) $\mathrm{C09}$

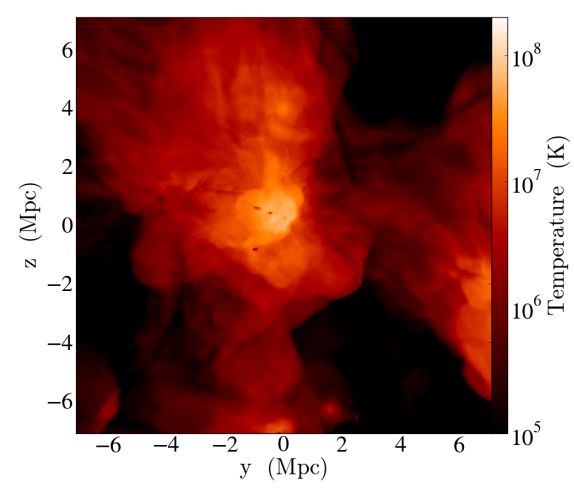

(1) $\mathrm{C} 12$

Fig. A.2. Temperature $x$-projections of all 12 clusters. All plots show the inner $5 \mathrm{Mpc} / \mathrm{h}$ and use the same color scheme, see Fig. 1. 


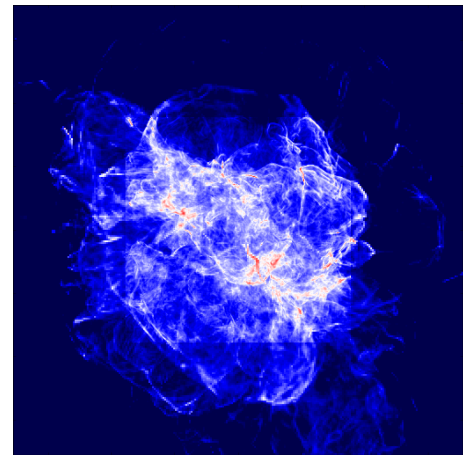

(a) $\mathrm{C} 01$

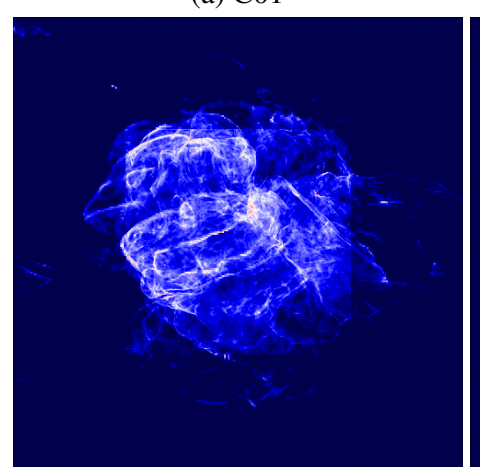

(d) $\mathrm{C} 04$

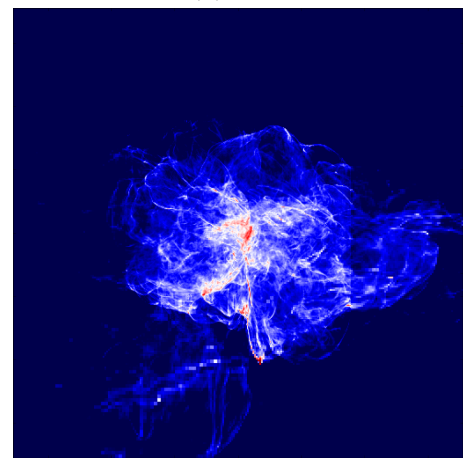

(g) $\mathrm{C} 07$

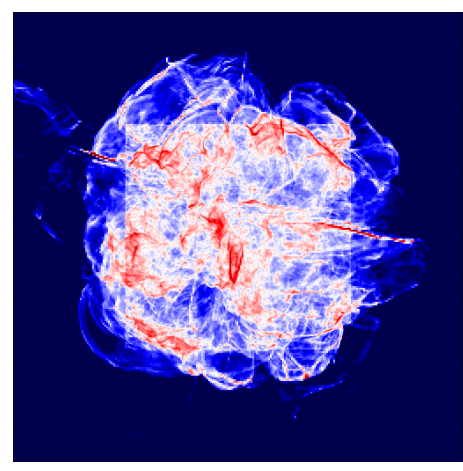

(j) $\mathrm{C} 10$

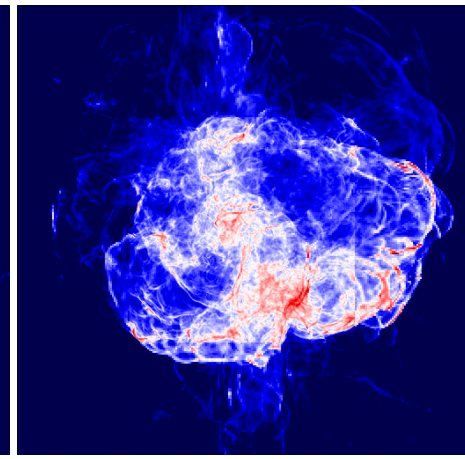

(b) $\mathrm{C} 02$

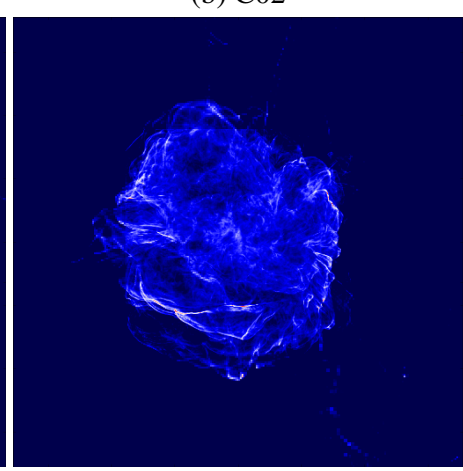

(e) $\mathrm{C} 05$

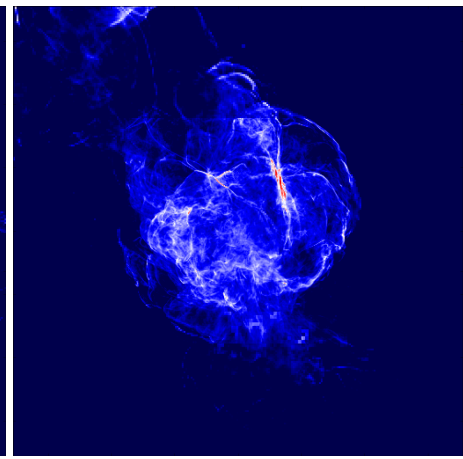

(h) $\mathrm{C} 08$

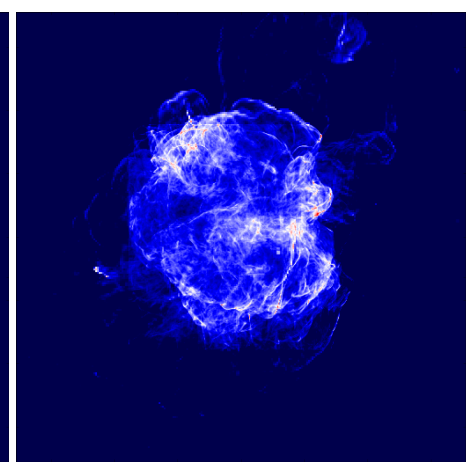

(k) $\mathrm{C} 11$

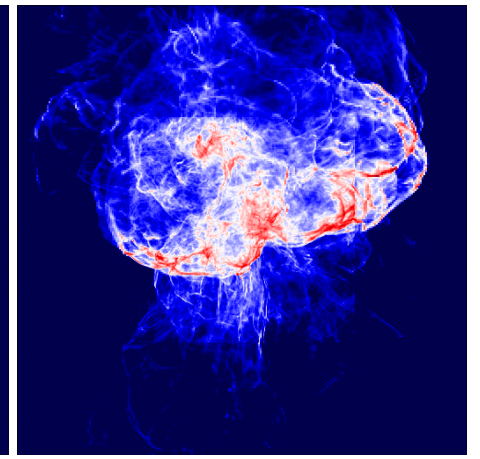

(c) $\mathrm{C} 03$

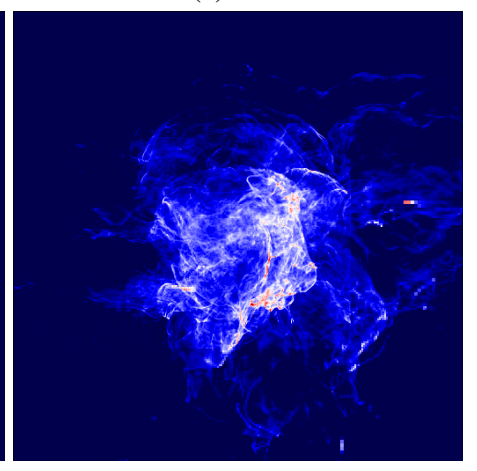

(f) $\mathrm{C} 06$

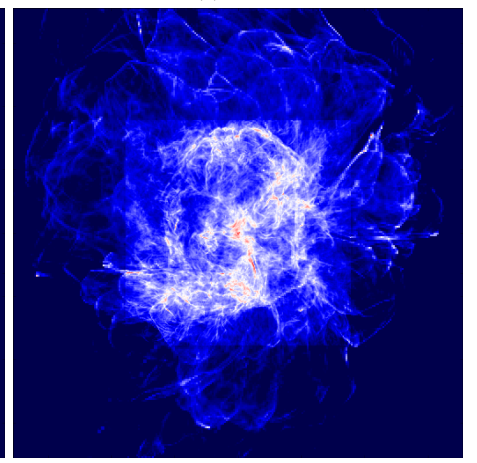

(i) $\mathrm{C} 09$

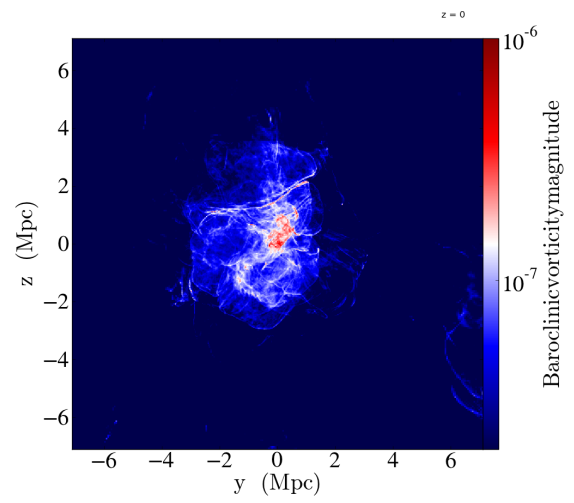

(1) $\mathrm{C} 12$

Fig. A.3. Baroclinic vorticity magnitude $x$-projections of all 12 clusters. All plots show the inner $5 \mathrm{Mpc} / \mathrm{h}$ and use the same color scheme, see Fig. l. The visibility of the grid refinement boundary is due to the usage of a common stencil when calculating the gradient or rotation on the grid data. 
H. Höller et al.: The distribution of metals in the ICM

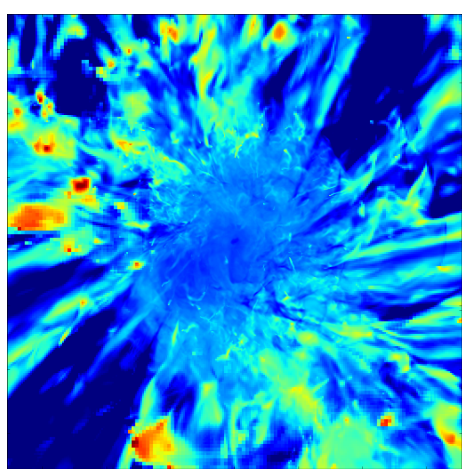

(a) $\mathrm{C} 01$

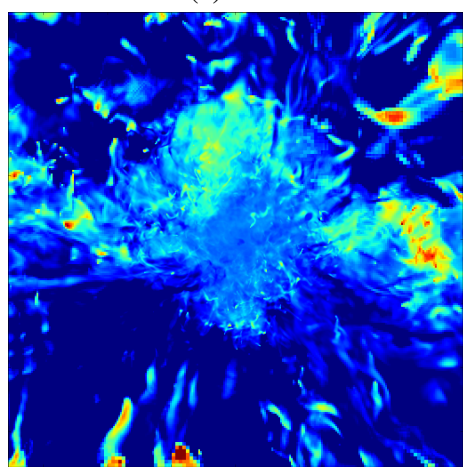

(d) $\mathrm{C} 04$

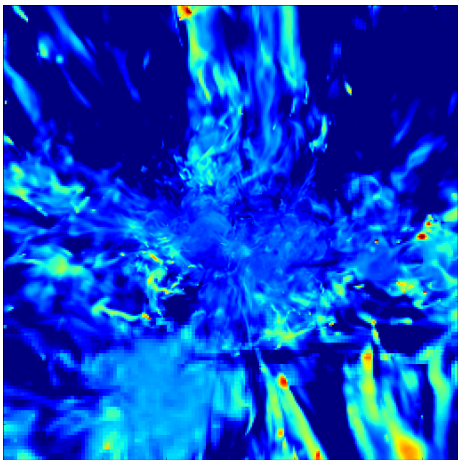

(g) $\mathrm{C} 07$

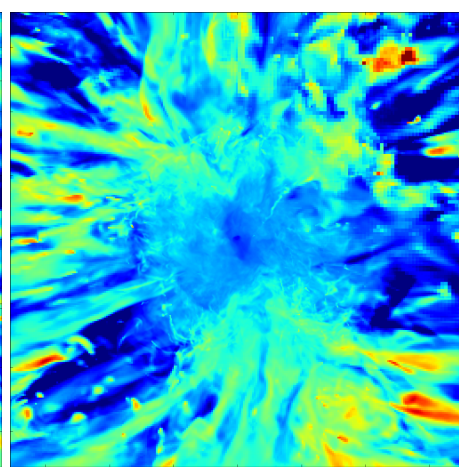

(b) $\mathrm{C} 02$

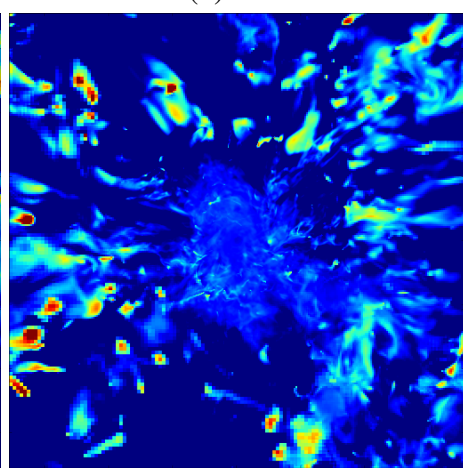

(e) $\mathrm{C} 05$

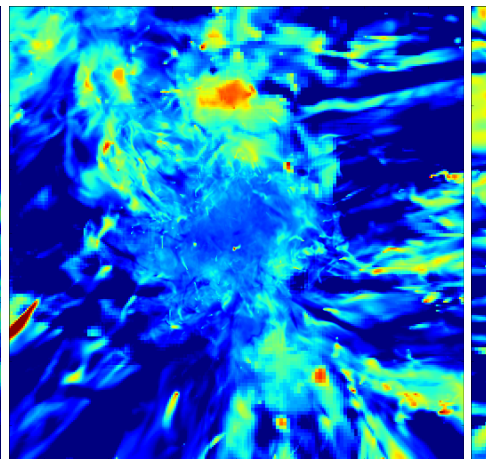

(h) $\mathrm{C} 08$

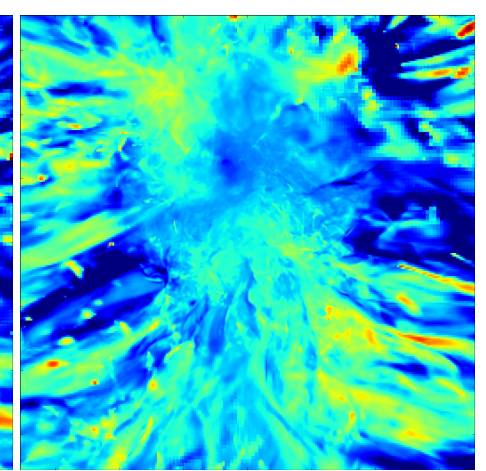

(c) $\mathrm{C} 03$

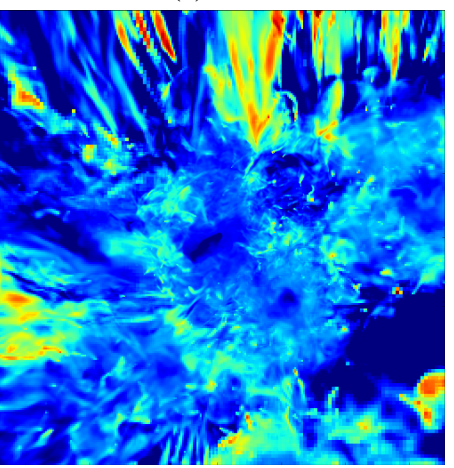

(f) $\mathrm{C} 06$

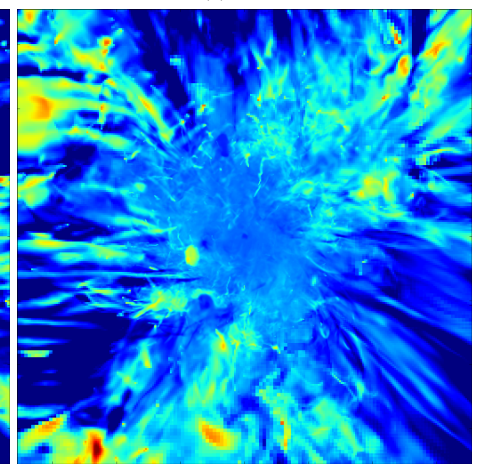

(i) $\mathrm{C} 09$

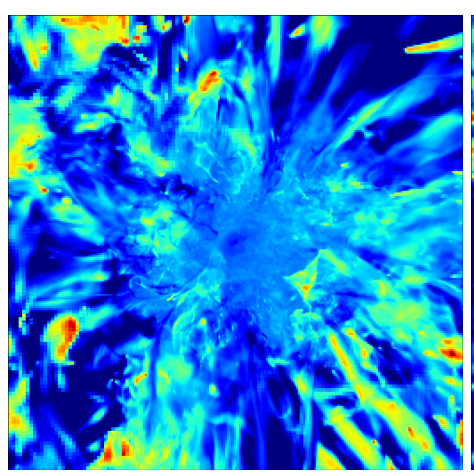

(j) $\mathrm{C} 10$

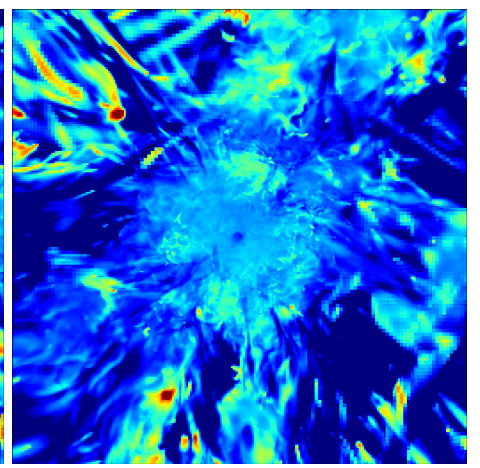

(k) $\mathrm{C} 11$

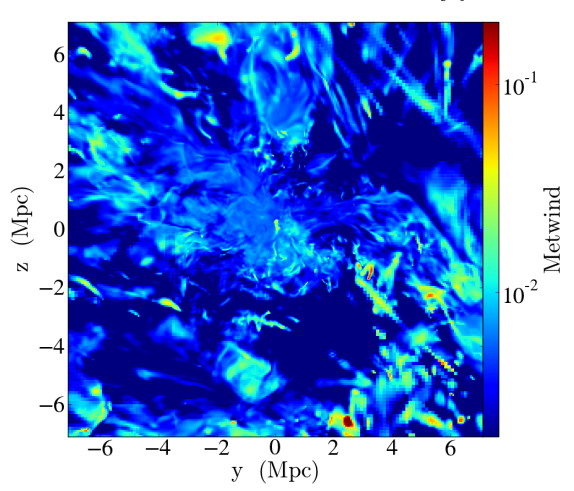

(1) $\mathrm{C} 12$

Fig. A.4. X-ray emissivity-weighted galactic wind metal mass-fraction $x$-projection of all 12 clusters. All plots show the inner $5 \mathrm{Mpc} / \mathrm{h}$ and use the same color scheme, see Fig. 1. 


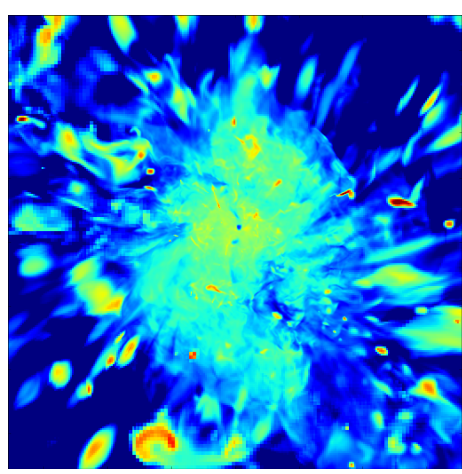

(a) $\mathrm{C} 01$

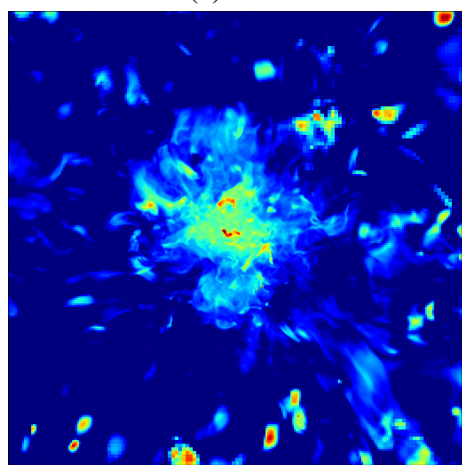

(d) $\mathrm{C} 04$

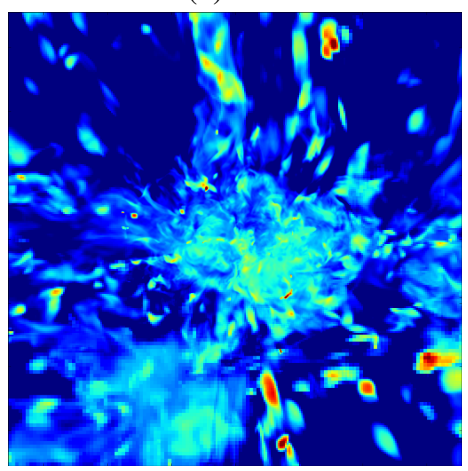

(g) $\mathrm{C} 07$

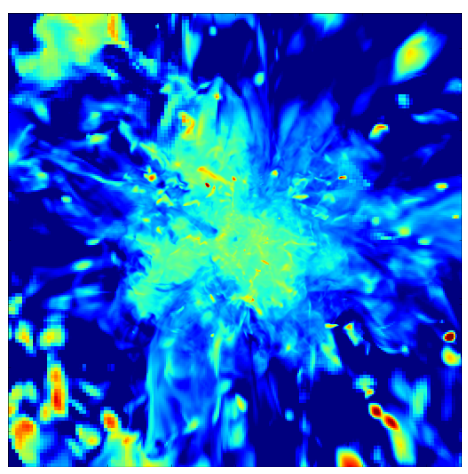

(j) $\mathrm{C} 10$

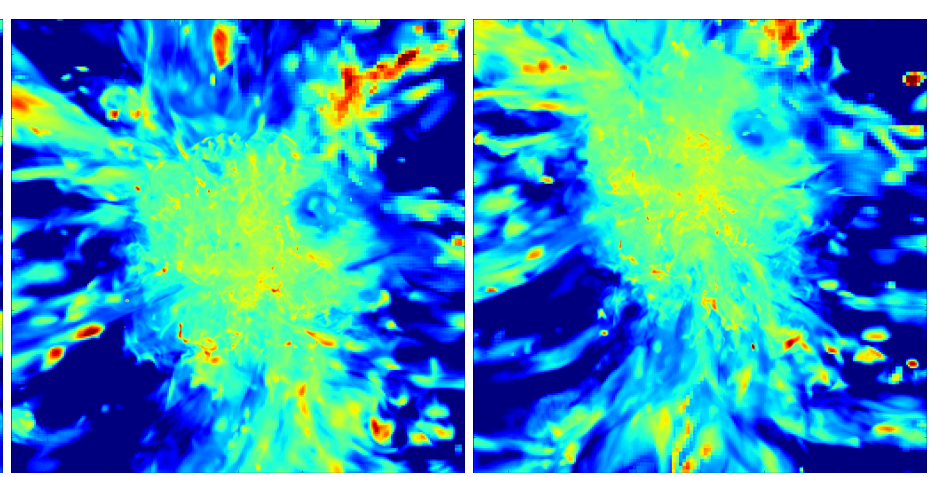

(c) $\mathrm{C} 03$

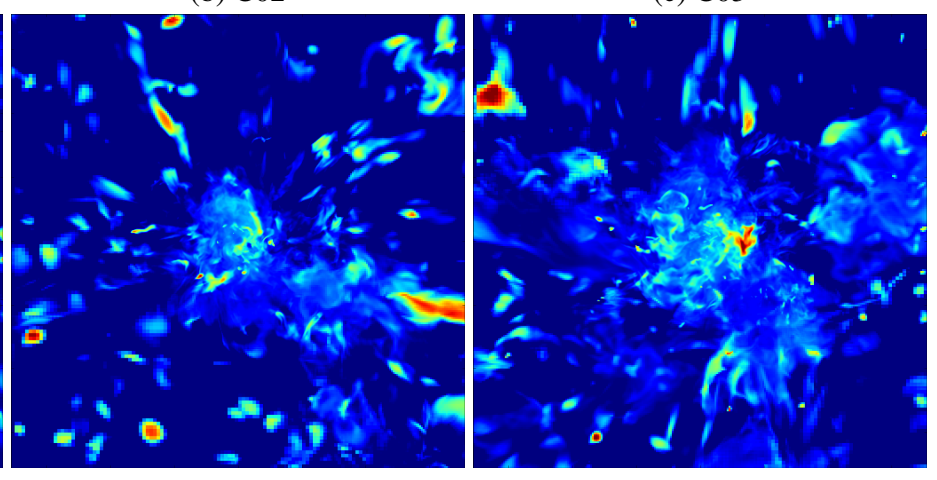

(e) $\mathrm{C} 05$

(f) $\mathrm{C} 06$

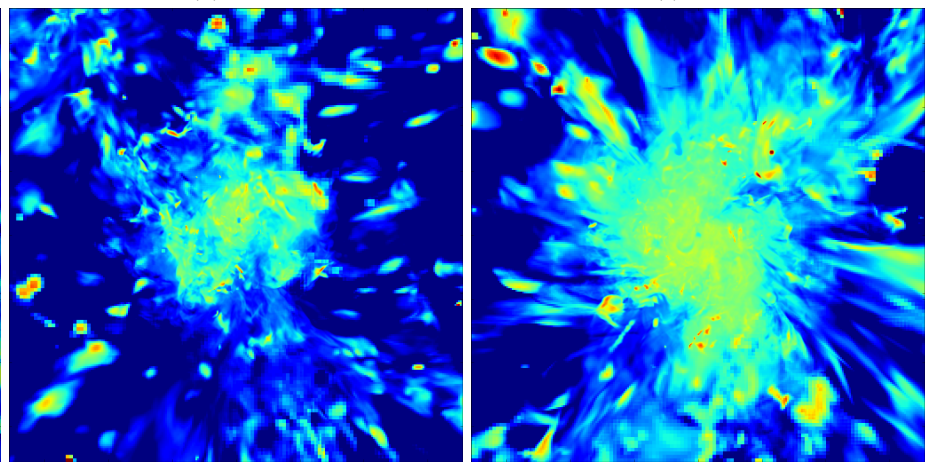

(h) $\mathrm{C} 08$

(i) $\mathrm{C} 09$

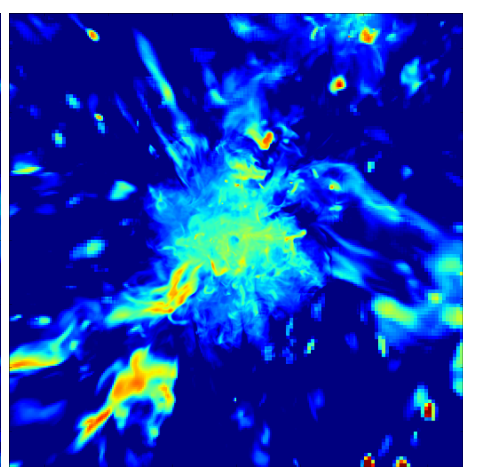

(k) $\mathrm{C} 11$

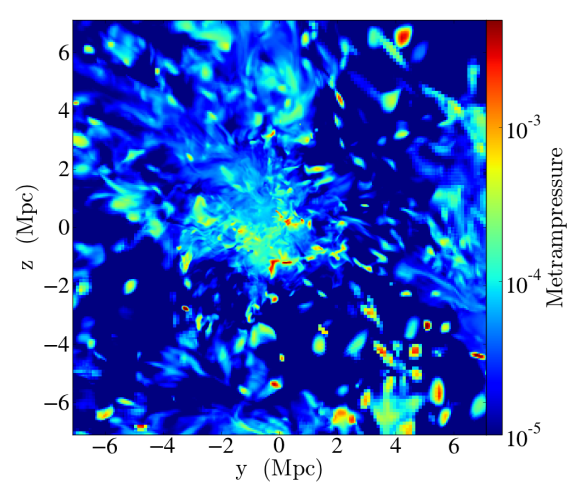

(1) $\mathrm{C} 12$

Fig. A.5. X-ray emissivity-weighted ram-pressure-stripped metal mass-fraction $x$-projection of all 12 clusters. All plots show the inner $5 \mathrm{Mpc} / \mathrm{h}$ and use the same color scheme, see Fig. 1. 
H. Höller et al.: The distribution of metals in the ICM

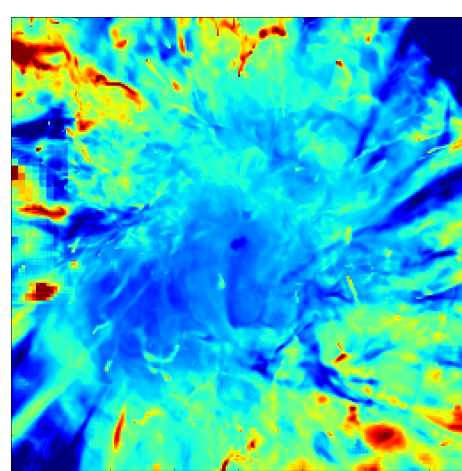

(a) $\mathrm{C} 01$

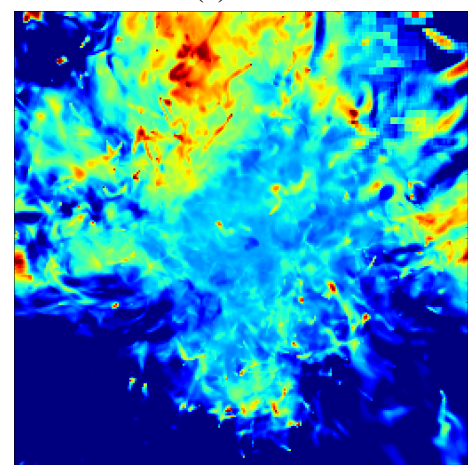

(d) $\mathrm{C} 04$

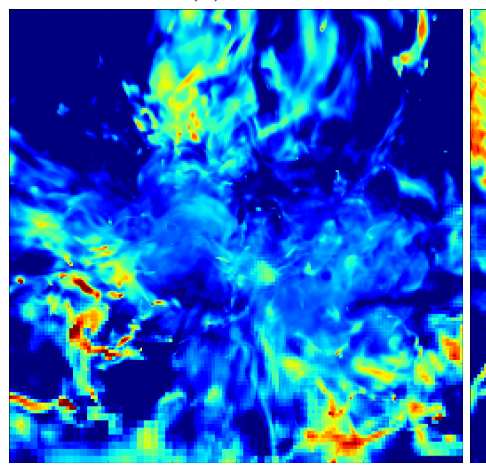

(g) $\mathrm{C} 07$

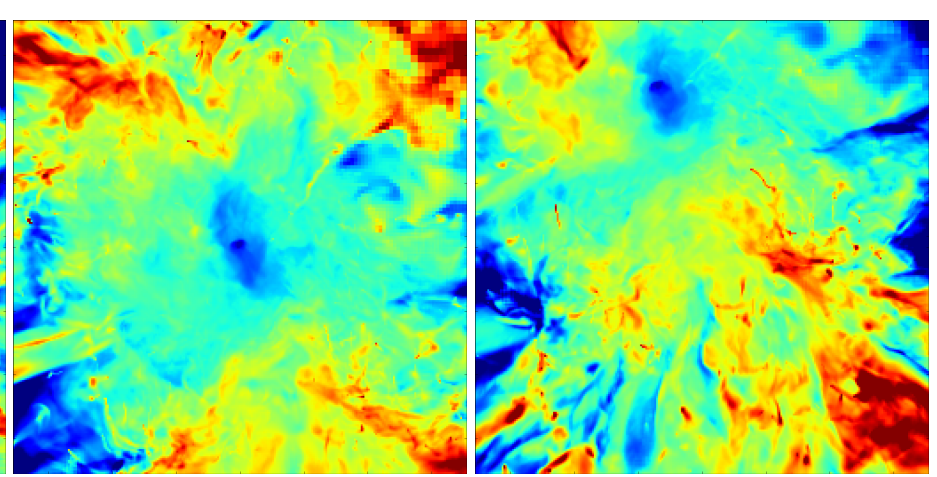

(b) $\mathrm{C} 02$

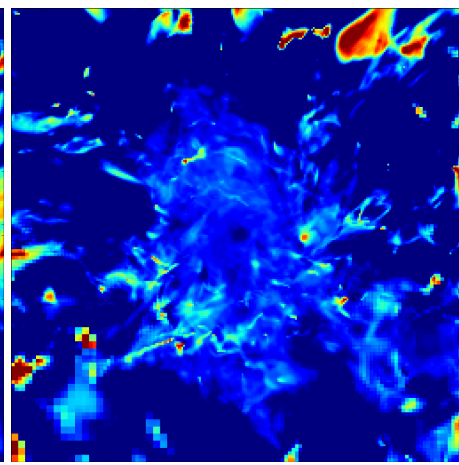

(e) $\mathrm{C} 05$

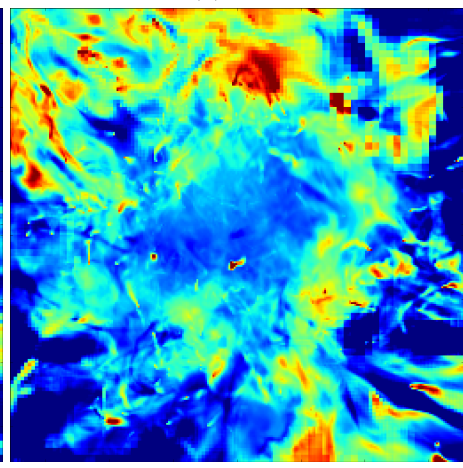

(h) $\mathrm{C} 08$ (c) $\mathrm{C} 03$

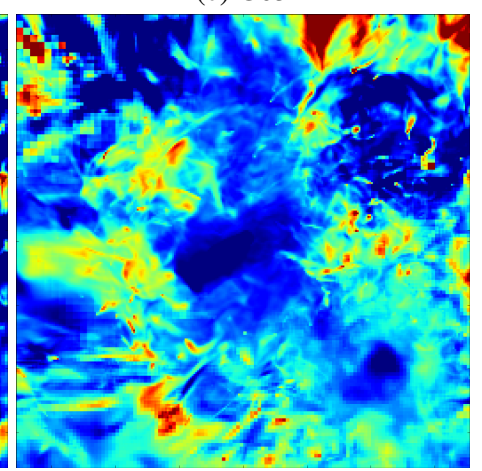

(f) $\mathrm{C} 06$

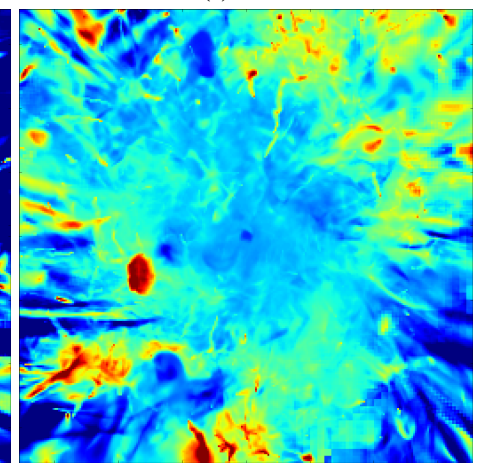

(i) $\mathrm{C} 09$

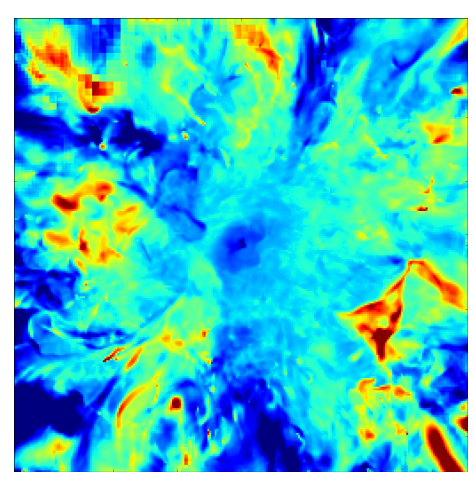

(j) $\mathrm{C} 10$

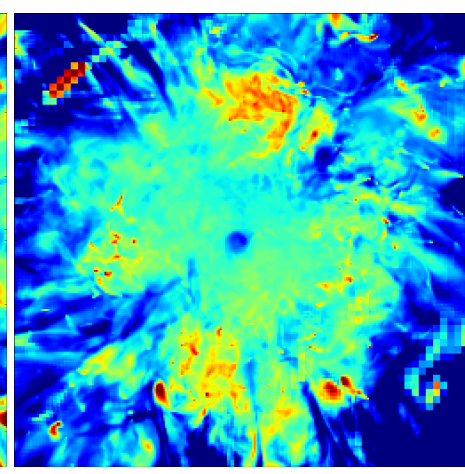

(k) $\mathrm{C} 11$

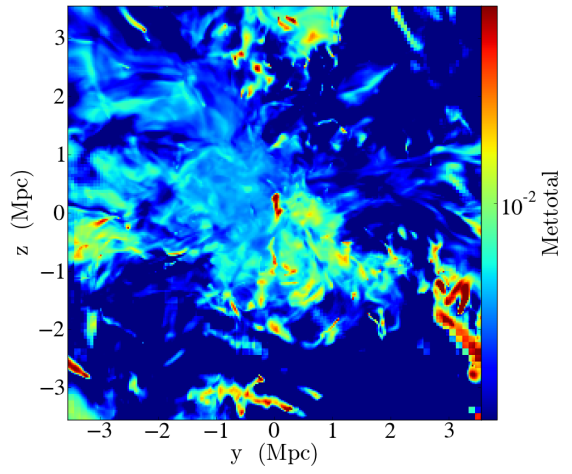

(1) $\mathrm{C} 12$

Fig. A.6. X-ray emissivity-weighted total metal mass-fraction $x$-projection of all 12 clusters. All plots show the inner $2.5 \mathrm{Mpc} / \mathrm{h}$ and use the same color scheme, see Fig. 1 . The color scheme represents a range from $0.2-2 Z_{\odot}$. 